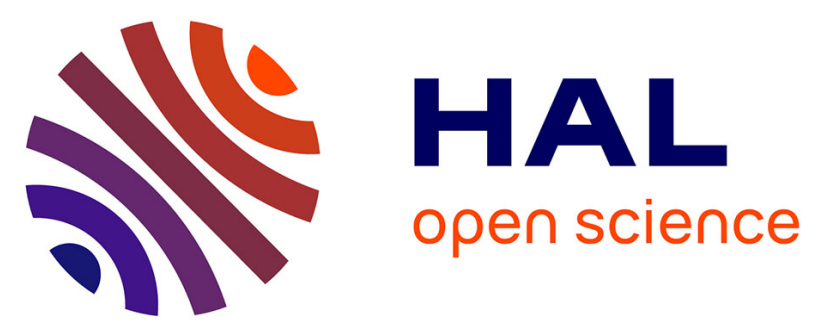

\title{
Vectorization of agrochemicals: amino acid carriers are more efficient than sugar carriers to translocate phenylpyrrole conjugates in the Ricinus system
} Hanxiang Wu, Sophie Marhadour, Zhi-Wei Lei, Wen Yang, Cécile Marivingt-Mounir, Jean-Louis Bonnemain, Jean-François Chollet

\section{To cite this version:}

Hanxiang Wu, Sophie Marhadour, Zhi-Wei Lei, Wen Yang, Cécile Marivingt-Mounir, et al.. Vectorization of agrochemicals: amino acid carriers are more efficient than sugar carriers to translocate phenylpyrrole conjugates in the Ricinus system. Environmental Science and Pollution Research, 2018, 25 (15), pp.14336-14349. 10.1007/s11356-016-8107-x . hal-02165243

\section{HAL Id: hal-02165243 \\ https://hal.science/hal-02165243}

Submitted on 25 Jun 2019

HAL is a multi-disciplinary open access archive for the deposit and dissemination of scientific research documents, whether they are published or not. The documents may come from teaching and research institutions in France or abroad, or from public or private research centers.
L'archive ouverte pluridisciplinaire HAL, est destinée au dépôt et à la diffusion de documents scientifiques de niveau recherche, publiés ou non, émanant des établissements d'enseignement et de recherche français ou étrangers, des laboratoires publics ou privés. 


\section{Vectorisation of agrochemicals: amino acid carriers are more efficient than sugar carriers to translocate phenylpyrrole conjugates in the Ricinus system}

Hanxiang $\mathrm{Wu}^{\mathrm{a}, \mathrm{c}, \mathrm{w}}$, Sophie Marhadour ${ }^{\mathrm{a}, \mathrm{w}}$, Zhi-Wei Lei ${ }^{\mathrm{b}}$, Wen Yang ${ }^{\mathrm{b}}$, Cécile MarivingtMounir $^{\mathrm{a}}$, Jean-Louis Bonnemain ${ }^{\mathrm{c}}$ and Jean-François Chollet ${ }^{\mathrm{a}, *}$

${ }^{a}$ Institut de Chimie des Milieux et des Matériaux de Poitiers (IC2MP), Unité Mixte de Recherche CNRS 7285, Université de Poitiers, 4 rue Michel Brunet, TSA 51106, F-86073 Poitiers cedex 9, France

${ }^{b}$ Guizhou Tea Reasearch Institute, Guizhou Academy of Agricultural Science, Guiyang, Guizhou 550009, China.

${ }^{c}$ Laboratoire Écologie et Biologie des Interactions, Unité Mixte de Recherche CNRS 7267, Université de Poitiers, 3 rue Jacques Fort, TSA 51106, F-86073 Poitiers Cedex 9, France

* Corresponding author: Tel. fax: +33 549453965

E-mail address: jean.franc.chollet@univ-poitiers.fr

$\Psi$ These authors contributed equally to the paper

This work was presented at the $46^{\text {th }}$ Congress of the "Groupe Français des Pesticides" which was held in Bordeaux from 17 to 19 May 2016 


\begin{abstract}
Producing quality food in sufficient quantity while using less agrochemical inputs will be one of the great challenges of the $21^{\text {st }}$ century. One way of achieving this goal is to greatly reduce the doses of plant protection compounds by improving the targeting of pests to eradicate. Therefore, we developed a vectorization strategy to confer phloem mobility to fenpiclonil, a contact fungicide from the Phenylpyrrole family used as a model molecule. It consists in coupling the antifungal compound to an amino acid or a sugar, so that the resulting conjugates are handled by the active nutrient transport systems. The method of click chemistry was used to synthesize three conjugates combining fenpiclonil to glucose or glutamic acid with a spacer containing a triazole ring. Systemicity tests with the Ricinus model have shown that the amino acid promoiety was clearly more favourable to phloem mobility than that of glucose. In addition, the transport of the amino acid conjugate is carrier mediated since the derivative of the L series was about 5 times more concentrated in the phloem sap than its counterpart of the D series. The systemicity of the L-derivative is $\mathrm{pH}$ dependent and almost completely inhibited by the protonophore CCCP. These data suggest that the phloem transport of the L-derivative is governed by a stereospecific amino acid carrier system energized by the proton motive force.
\end{abstract}

Keywords Phenylpyrroles, fungicide, systemicity, phloem transport, sustainable agriculture, carrier mediated 


\section{Introduction}

Because of the growing public concern about environmental and health hazards, there has been a clear trend of reducing pesticide use worldwide. On the other hand, reducing pesticide use is required not to affect the quantity and quality of agricultural products (Chollet et al. 2014). Therefore, how to increase the pesticide efficiency will be a great challenge for the agricultural chemicals industry. A serious problem is that only a very small part of pesticides (less than $0.1 \%$ ) actually reaches the sites of action, and off-target portion becomes environmental pollutant (Wang and Liu 2007).

To keep the dose at the lowest possible level and enhance bioavailability are prime properties to both agrochemical and drug new active ingredients. As commonly defined, bioavailability implies the extent and rate at which a drug becomes available in the systemic circulation (Mannhold et al. 2009). This term can also be used in agrochemical research to describe a pesticide which exerts their biological action in certain plant organs or cells at distance from the site of uptake. Both agrochemicals and drugs require high bioavailability and targeted delivery to ensure desirable localization at the site of action while limiting toxicity in nontarget organs or organisms.

The prodrug concept, as first introduced by Adrian Albert in 1958 (Albert 1958), can be used to improve the physicochemical properties or bioproperties of the parent drug molecule in such a way as to enhance its deliverability (Stella et al. 2007). Prodrug strategies have been used to solve the problems in oral absorption, organ distribution, metabolism, and excretion (Zawilska et al. 2013). One of the promising means of prodrug strategies is carrier-mediated transport (Sugano et al. 2010). Drug transporters are now widely acknowledged as important determinants governing drug absorption, excretion, and, in many cases, extent of drug entry into target organs (DeGorter et al. 2012). The frequently exploited transporters include peptide, amino acid, and glucose transporters (Fan et al. 2011, Giacomini et al. 2010, Gynther et al. 2008).

Carrier-mediated processes in pesticide uptake and transport is still in the initial stage. It has been known for a long time that phloem mobile pesticides are needed to control root or vascular diseases (Chollet et al. 2005, Edgington 1981), as well as piercing and sucking insects. For example, phloem mobile insecticides (Hu et al. 2010, Yang et al. 2011, Wu et al 2012) can be a necessary complement to biological control, cultural practices and genetic resistance utilization in controlling aphids (Bonnemain 2010, Dedryver et al. 2010, Dogimont et al. 2010). Phloem systemicity of agrochemicals can be restricted by their inability to be 
transported across the plasma membrane from apoplast to symplast. Over the last two decades, there have been a few attempts to improve pesticide phloem mobility or transport to root system utilizing membrane sugar or amino acid transporters (Chollet et al. 1997, Delétage-Grandon et al. 2001, Wu et al. 2012).

The purpose of the present work was to select an ideal promoiety for designing phloem systemic profungicide. Fenpiclonil is a phenylpyrrole fungicide based on chemical modifications of a bacterial metabolite pyrrolnitrin (van Pee and Ligon 2000). This nonsystemic fungicide was selected as a model parent compound to test our vectorisation strategy and at first to compare the ability of amino acid and sugar carrier systems to translocate large chlorinated xenobiotics. Three designed fenpiclonil conjugates were synthesized via click chemistry.

\section{Material and methods}

\section{Synthesis}

Some reactions were carried out under nitrogen. All reactions were monitored by TLC analysis using Merck silica gel 60F-254 thin-layer plates. Column chromatography was carried out on silica gel Merck $60(0.015-0.04 \mathrm{~mm})$. Melting points were determined on an Electrothermal IA 9200 melting point apparatus and are uncorrected. Optical rotations were measured at $20^{\circ} \mathrm{C}$ in a $1 \mathrm{~cm}$ cell in the stated solvent; $[\alpha]^{20} \mathrm{D}$ values are given in $10^{-1}$ deg. $\mathrm{cm}^{2} \cdot \mathrm{g}^{-1}$ (concentration $c$ given as $\mathrm{g} / 100 \mathrm{~mL}$ ). ${ }^{1} \mathrm{H}$ and ${ }^{13} \mathrm{C}$ NMR spectra were performed in DMSO-d6 using a Bruker AVANCE $400 \mathrm{MHz}$ spectrometer. DEPT 135 and ${ }^{1} \mathrm{H}-{ }^{13} \mathrm{C}$ experiments were used to confirm the NMR peak assignements. Chemical shifts are reported as $\delta$ values in parts per million (ppm) relative to tetramethylsilane as internal standard and coupling constants $(J)$ are given in hertz $(\mathrm{Hz})$. The following abbreviations are used to describe peak patterns when appropriate : s (singlet), d (doublet), $t$ (triplet), q (quartet), $\mathrm{m}$ (multiplet). High-resolution mass spectra were obtained on a Bruker Qtof Maxis Impact spectrometer.

\section{Plant material}

Castor bean seeds (Ricinus communis L. cv Sanguineus), obtained from Graines Girerd et Fils (Le Thor, France) were placed in wet cotton wool for $24 \mathrm{~h}$ at $27^{\circ} \mathrm{C} \pm 1{ }^{\circ} \mathrm{C}$ prior to sowing in 
vermiculite watered with tap water. Seedlings were grown in a humid atmosphere $(80 \% \pm$ $5 \%)$ at $27^{\circ} \mathrm{C} \pm 1{ }^{\circ} \mathrm{C}$.

\section{Phloem sap collection and analysis}

The sap collection method was similar to that already described (Rocher et al. 2006). The phloem sap was analyzed by HPLC after dilution with UHQ grade water $(1+9 \mathrm{v} / \mathrm{v})$. We employed reversed phase chromatography using an Ascentis Express RP-amide C16 column (length $250 \mathrm{~mm}$, internal diameter $4.6 \mathrm{~mm}, 5 \mu \mathrm{M}$ ) (Supelco, Bellefonte, PA) in accordance with the procedure set out in Table I. Results were processed with PC 1000 software v3.5 from Thermo Fisher Scientific (Courtabœuf, France).

\section{Chemicals}

The compounds to be added to incubation solutions were from Acros Organics (Noisy-leGrand, France) (4-morpholinoethanesulfonic acid [MES], 2-[4-(2-hydroxyethyl)-1piperazine] ethanesulfonic acid [HEPES]), from Alfa-Aesar (Karlsruhe, Germany) (Carbonyl cyanide 3-chlorophenylhydrazone [CCCP]).

\section{Physicochemical properties}

Physicochemical properties and descriptors were predicted using ACD / Labs Percepta 2015 release (Build 2726) software from Advanced Chemistry Development, Inc. (Toronto, Canada). The calculated properties (Table II) were chosen according to their influence on passive membrane transport in plants (Rocher et al. 2016).

\section{Results and discussion}

\section{Synthesis of glucose and amino acid conjugates}

Fenpiclonil was the first phenylpyrrole fungicide developed by Ciba-Geigy at the end of the eighties for seed treatment in cereals (Nyfeler and Ackermann 1992). It is an analogue of the antifungal metabolite pyrrolnitrin, which is produced by several Pseudomoniae. Although no longer commercialized, fenpiclonil was selected as a model compound because of the numerous possibilities of adding substituents at various sites in the molecule, in particular to 
confer systemicity to this non mobile fungicide. These substituents can be carboxylic groups to exploit the ion trap mechanism (Chollet et al. 2004, 2005).

In this work, we chose another strategy which consists in synthesizing conjugates associating the fungicide and a nutrient and in testing if the resulting compounds are recognized and manipulated by an active carrier. We have developed and validated this concept at first with an herbicide and an amino acid (Chollet et al. 1997, Delétage-Grandon et al. 2001, Dufaud et al. 1994). Then, this strategy was extended to an insecticide with a sugar moiety (Hu et al. 2010, Yang et al. 2011).

In order to possibly promote the interaction between the amino acid or the sugar and their respective carriers, a spacer was introduced between the fungicide and the nutrient. The selected spacer includes a 1,2,3-triazole ring linked to the antifungal compound with an amid bond. The 1,2,3-triazole ring has the advantage of synthesizing easily with good yields by « click chemistry » process. In 2001, Sharpless and coworkers introduce the concept of « click chemistry » in order to develop a wide range of compounds for a large scope of applications (Kolb et al. 2001). Several types of reaction fulfill all the criteria like simplicity to perform with high yield, stereospecificity and easy removing of byproducts. The classic Huisgen azide-alkyne 1,3-dipolar cycloaddition led to mixtures of the two regioisomers (Huisgen et al. 1965) whereas the copper-catalyzed reaction named CuAAC (« Copper(I)catalyzed Azide-Alkyne Cycloaddition ») allows the synthesis of the 1,4-disubstituted regioisomers specifically (Rostovtsev et al. 2002, Tornoe et al. 2002).

In this way for the synthesis of compounds 12 (conjugate with B-D-glucose), L-13 and D-13 (conjugates with L- or D- glutamic acid), the following multi-step synthesis was established according to figure 1.

\section{Synthesis of azido derivatives from protected sugar or amino acids (Fig. 1 ; compounds 2, 6, 8)}

We first started with the synthesis of azido derivatives $(2, \mathbf{6}, \mathbf{8})$ from commercially available protected sugar or amino acid. Pentaacetate glucose was reacted with azidotrimethylsilane in the presence of tin (IV) chloride to afford glucose derivative 2 with $86 \%$ yield (Cagnoni et al. 2011). We next decided to prepare azido derivatives from protected glutamic acid. The first step consisted of the synthesis of 2-azidoethanamine 4 which was obtained with $83 \%$ yield by nucleophilic substitution of sodium azide on 2-bromoethylamine hydrobromide $\mathbf{1}$ in water at $75^{\circ} \mathrm{C}$ (Mayer and Maier 2007). Then 2-azidoethanamine 4 was condensed with protected Lor D- glutamic acid derivatives (respectively compounds 5 and 7) in the presence of 4- 
dimethylaminopyridine (DMAP) and using the condensing agent, 1-(3dimethylaminopropyl)-3-ethylcarbodiimide hydrochloride (EDCl) (Sheehan et al. 1961). This latter allowed to set an amide bond between the amine function of the compound $\mathbf{4}$ and the carboxylic acid function of the compounds 5 or 7 (Sheehan and Hess 1955). The compounds 6 and $\mathbf{8}$ were obtained from moderate to good yields, $81 \%$ and $43 \%$ respectively.

-Azido-6-(ethanoyloxymethyl)tetrahydro-2H-pyran-3,4,5-triyl triethanoate (compound 2). To a solution of glucose pentaacetate $(5.9 \mathrm{~g}, 15.0 \mathrm{mmol}, 1$ equiv) and azidotrimethylsilane (2.98 mL, $22.5 \mathrm{mmol}, 1.5$ equiv) in anhydrous dichloromethane $(150 \mathrm{~mL})$ was added dropwise tin (IV) chloride (1.95 $\mathrm{mL}, 16.5 \mathrm{mmol}, 1.1$ equiv). The reaction mixture was stirred at room temperature for $2 \mathrm{~h}$ and then washed with sodium bicarbonate and water. The organic layer was dried over $\mathrm{MgSO}_{4}$, filtered, and concentrated under vacuum. The crude product was purified by silica gel column chromatography using pentane/ethyl acetate $(8: 2)$ as eluent to afford compound 2 as a white powder ( $4.9 \mathrm{~g}, 86 \%$ yield).

$\mathrm{Rf}=0.62$ (pentane/ethyl acetate: $6 / 4) ; \mathrm{Mp}=128-129^{\circ} \mathrm{C}$ (lit. mp $\left.129^{\circ} \mathrm{C}\right) .{ }^{1} \mathrm{H}$ NMR $(400$ MHz, DMSO-d $\left.d_{6}\right): \delta 5.37\left(\mathrm{t}, 1 \mathrm{H},{ }^{3} J={ }^{3} J^{\prime}=9.3 \mathrm{~Hz}, \mathrm{H}_{\mathrm{c}}\right), 5.18\left(\mathrm{~d}, 1 \mathrm{H},{ }^{3} J=9.3 \mathrm{~Hz}, \mathrm{H}_{\mathrm{a}}\right), 5.02(\mathrm{t}$, $\left.1 \mathrm{H},{ }^{3} J={ }^{3} J^{\prime}=9.3 \mathrm{~Hz}, \mathrm{H}_{\mathrm{d}}\right), 4.86\left(\mathrm{t}, 1 \mathrm{H},{ }^{3} J={ }^{3} J^{\prime}=9.3 \mathrm{~Hz}, \mathrm{H}_{\mathrm{b}}\right), 4.23-4.10\left(\mathrm{~m}, 3 \mathrm{H}, \mathrm{H}_{\mathrm{e}}\right.$ and $\left.\mathrm{H}_{\mathrm{f}}\right)$, 2.08, 2.07, 2.03, $1.99\left(4 \mathrm{~s}, 12 \mathrm{H}, \mathrm{H}_{\mathrm{g}}\right) .{ }^{13} \mathrm{C} \mathrm{NMR}\left(100 \mathrm{MHz}, \mathrm{DMSO}-d_{6}\right): \delta 170.03(\mathrm{C}=\mathrm{O})$, $169.54(\mathrm{C}=\mathrm{O}), 169.25(\mathrm{C}=\mathrm{O}), 169.12(\mathrm{C}=\mathrm{O}), 86.23\left(\mathrm{C}_{\mathrm{a}}\right), 72.76\left(\mathrm{C}_{\mathrm{e}}\right), 71.77\left(\mathrm{C}_{\mathrm{c}}\right), 70.22\left(\mathrm{C}_{\mathrm{b}}\right)$, $67.75\left(\mathrm{C}_{\mathrm{d}}\right), 61.68\left(\mathrm{C}_{\mathrm{f}}\right), 20.47,20.34,20.28,20.23\left(4 \mathrm{C}_{\mathrm{g}}\right)$. HRMS (ESI, $\left.\mathrm{CH}_{3} \mathrm{CN}\right): \mathrm{m} / z$ calcd for $\mathrm{C}_{14} \mathrm{H}_{19} \mathrm{~N}_{3} \mathrm{O}_{9}[\mathrm{M}+\mathrm{Na}]^{+} 396.1019, \mathrm{~m} / z$ found 396.1013.

\section{- Azidoethanamine (compound 4).}

To a solution of 2-bromoethylamine hydrobromide 3 (20.4 g, $100.0 \mathrm{mmol}, 1$ equiv) in water $(142 \mathrm{~mL})$ was added sodium azide (14.2 $\mathrm{g}, 220 \mathrm{mmol}, 2.2$ equiv). The reaction mixture was heated at $75^{\circ} \mathrm{C}$ for $18 \mathrm{~h}$. After cooling at room temperature, sodium hydroxide $(4.0 \mathrm{~g}, 100.0$ mmol, 1 equiv) was added portionwise to the reaction mixture. The aqueous layer was extracted twice with dichloromethane. The combined organic layers were dried over $\mathrm{MgSO}_{4}$, filtered and concentrated under vacuum (without heating) to afford compound $\mathbf{4}$ as a colourless oil ( $7.1 \mathrm{~g}, 83 \%$ yield). This material was used in the next reaction without further purification. 
$\mathrm{Rf}=0.39$ (ethyl acetate/methanol: 6/4). ${ }^{1} \mathrm{H}$ NMR (400 MHz, $\left.\mathrm{CDCl}_{3}\right): \delta 3.36\left(\mathrm{t}, 2 \mathrm{H},{ }^{3} J=5.7\right.$

$\left.\mathrm{Hz}, \mathrm{H}_{\mathrm{a}}\right), 2.88\left(\mathrm{t}, 2 \mathrm{H},{ }^{3} \mathrm{~J}=5.7 \mathrm{~Hz}, \mathrm{H}_{\mathrm{b}}\right), 1.24\left(\mathrm{~s}, 2 \mathrm{H}, \mathrm{NH}_{2}\right) .{ }^{13} \mathrm{C} \mathrm{NMR}\left(100 \mathrm{MHz}, \mathrm{CDCl}_{3}\right): \delta$

$54.85\left(\mathrm{C}_{\mathrm{a}}\right), 41.54\left(\mathrm{C}_{\mathrm{b}}\right)$.

- Tert-butyl 5-(2-azidoethylamino)-2-(tert-butoxycarbonylamino)-5-oxopentanoate (compound 6).

To a solution of $N$-Boc- $L$-glutamic acid 1-tert-butyl ester 5 (4.55 g, $15.0 \mathrm{mmol}, 1$ equiv) in anhydrous dichloromethane $(45 \mathrm{~mL})$ cooled to $0^{\circ} \mathrm{C}$ was added compound 4 (2.58 g, 30.0 mmol, 2 equiv), 1-(3-dimethylaminopropyl)-3-ethylcarbodiimide hydrochloride (6.04 g, 31.5 mmol, 2.1 equiv) and 4-dimethylaminopyridine (183 mg, $1.50 \mathrm{mmol}, 0.1$ equiv). The reaction mixture was purged with nitrogen through the septum and then stirred at $0{ }^{\circ} \mathrm{C}$ for $2 \mathrm{~h}$. The mixture was allowed to reach room temperature and then stirred for $18 \mathrm{~h}$. Water was added and the organic layer was extracted twice with dichloromethane. The combined organic layers were washed with water, dried over $\mathrm{MgSO}_{4}$, filtered and concentrated under vacuum. The crude product was purified by silica gel column chromatography using pentane/ethyl acetate $(8: 2)$ as eluent to afford compound 6 as a colourless oil (4.5 g, $81 \%$ yield).

$\mathrm{Rf}=0.26$ (pentane/ethyl acetate: $6 / 4) .{ }^{1} \mathrm{H}$ NMR $\left(400 \mathrm{MHz}, \mathrm{DMSO}-d_{6}\right): \delta 8.10\left(\mathrm{t}, 1 \mathrm{H},{ }^{3} J=5.3\right.$ $\mathrm{Hz}, \mathrm{NH}), 7.15\left(\mathrm{~d}, 1 \mathrm{H},{ }^{3} J=7.8 \mathrm{~Hz}, \mathrm{NH}\right), 3.84-3.74\left(\mathrm{~m}, 1 \mathrm{H}, \mathrm{H}_{\mathrm{e}}\right), 3.38-3.24\left(\mathrm{~m}, 4 \mathrm{H}, \mathrm{H}_{\mathrm{a}}\right.$ and $\left.\mathrm{H}_{\mathrm{b}}\right)$, $2.18\left(\mathrm{t}, 2 \mathrm{H},{ }^{3} \mathrm{~J}=7.7 \mathrm{~Hz}, \mathrm{H}_{\mathrm{c}}\right), 1.97-1.71\left(\mathrm{~m}, 2 \mathrm{H}, \mathrm{H}_{\mathrm{d}}\right), 1.43\left(\mathrm{~s}, 9 \mathrm{H}, \mathrm{H}_{\mathrm{f}}\right), 1.42\left(\mathrm{~s}, 9 \mathrm{H}, \mathrm{H}_{\mathrm{g}}\right) .{ }^{13} \mathrm{C}$ NMR (100 MHz, DMSO- $\left.d_{6}\right): \delta 171.66(\mathrm{C}=\mathrm{O}), 171.56(\mathrm{C}=\mathrm{O}), 155.48(\mathrm{C}=\mathrm{O}), 80.25(\mathrm{C}), 78.01$ $(\mathrm{C}), 53.95\left(\mathrm{C}_{\mathrm{e}}\right), 49.93\left(\mathrm{C}_{\mathrm{a}}\right), 38.17\left(\mathrm{C}_{\mathrm{b}}\right), 31.61\left(\mathrm{C}_{\mathrm{c}}\right), 28.17\left(3 \mathrm{C}_{\mathrm{g}}\right), 27.62\left(3 \mathrm{C}_{\mathrm{f}}\right), 26.48\left(\mathrm{C}_{\mathrm{d}}\right)$. HRMS (ESI, $\mathrm{CH}_{3} \mathrm{CN}$ ): $\mathrm{m} / z$ calcd for $\mathrm{C}_{16} \mathrm{H}_{29} \mathrm{~N}_{5} \mathrm{O}_{5}[\mathrm{M}+\mathrm{Na}]^{+} 394.2066, \mathrm{~m} / z$ found 394.2061.

\section{-Benzyl 5-(2-azidoethylamino)-2-(tert-butoxycarbonylamino)-5-oxopentanoate (compound} 8).

To a solution of $N$-Boc- $D$-glutamic acid 1-benzyl ester 7 (10.1 g, $30.0 \mathrm{mmol}, 1$ equiv) in anhydrous dichloromethane $(103 \mathrm{~mL})$ cooled to $0{ }^{\circ} \mathrm{C}$ was added compound 4 (5.16 g, 60.0 mmol, 2 equiv), 1-(3-dimethylaminopropyl)-3-ethylcarbodiimide hydrochloride (12.1 g, 62.9 mmol, 2.1 equiv) and 4-dimethylaminopyridine ( $366 \mathrm{mg}, 3.00 \mathrm{mmol}, 0.1$ equiv). The reaction mixture was purged with nitrogen through the septum and then stirred at $0{ }^{\circ} \mathrm{C}$ for $2 \mathrm{~h}$. The mixture was allowed to reach room temperature and then stirred for $18 \mathrm{~h}$. Water was added and the organic layer was extracted twice with dichloromethane. The combined organic layers were washed with water, dried over $\mathrm{MgSO}_{4}$, filtered and concentrated under vacuum. The 
crude product was purified by silica gel column chromatography using pentane/ethyl acetate ( $7: 3)$ as eluent to afford the compound 8 as a white powder ( $5.2 \mathrm{~g}, 43 \%$ yield).

$\mathrm{Rf}=0.42$ (pentane/ethyl acetate: $6 / 4) ; \mathrm{Mp}=68-69^{\circ} \mathrm{C} .{ }^{1} \mathrm{H}$ NMR $\left(400 \mathrm{MHz}, \mathrm{DMSO}-d_{6}\right): \delta$ $8.10\left(\mathrm{t}, 1 \mathrm{H},{ }^{3} J=5.5 \mathrm{~Hz}, \mathrm{NH}\right), 7.43-7.34\left(\mathrm{~m}, 5 \mathrm{H}, \mathrm{H}_{\mathrm{h}}, \mathrm{H}_{\mathrm{i}}\right.$ and $\left.\mathrm{H}_{\mathrm{j}}\right), 6.97\left(\mathrm{~d}, 1 \mathrm{H},{ }^{3} J=8.1 \mathrm{~Hz}, \mathrm{NH}\right)$, $5.12\left(\mathrm{~s}, 2 \mathrm{H}, \mathrm{H}_{\mathrm{g}}\right), 4.00-3.95\left(\mathrm{~m}, 1 \mathrm{H}, \mathrm{H}_{\mathrm{e}}\right), 3.44-3.19\left(\mathrm{~m}, 4 \mathrm{H}, \mathrm{H}_{\mathrm{a}}\right.$ and $\left.\mathrm{H}_{\mathrm{b}}\right), 2.42\left(\mathrm{t}, 2 \mathrm{H},{ }^{3} J=7.7\right.$ $\left.\mathrm{Hz}, \mathrm{H}_{\mathrm{c}}\right), 2.00-1.76\left(\mathrm{~m}, 2 \mathrm{H}, \mathrm{H}_{\mathrm{d}}\right), 1.41\left(\mathrm{~s}, 9 \mathrm{H}, \mathrm{H}_{\mathrm{f}}\right) .{ }^{13} \mathrm{C}$ NMR (100 MHz, DMSO-d $\left.d_{6}\right): \delta 172.18$ $(\mathrm{C}=\mathrm{O}), 171.82(\mathrm{C}=\mathrm{O}), 155.28(\mathrm{C}=\mathrm{O}), 136.20(\mathrm{C}), 128.40\left(2 \mathrm{C}_{\mathrm{i}}\right), 127.95\left(\mathrm{C}_{\mathrm{j}}\right), 127.83\left(2 \mathrm{C}_{\mathrm{h}}\right)$, $78.12(\mathrm{C}), 65.43\left(\mathrm{C}_{\mathrm{g}}\right), 53.50\left(\mathrm{C}_{\mathrm{e}}\right), 49.93\left(\mathrm{C}_{\mathrm{a}}\right), 38.15\left(\mathrm{C}_{\mathrm{b}}\right), 30.10\left(\mathrm{C}_{\mathrm{c}}\right), 28.14\left(3 \mathrm{C}_{\mathrm{f}}\right), 27.09\left(\mathrm{C}_{\mathrm{d}}\right)$. HRMS (ESI, $\mathrm{CH}_{3} \mathrm{CN}$ ): $m / z$ calcd for $\mathrm{C}_{19} \mathrm{H}_{27} \mathrm{~N}_{5} \mathrm{O}_{5}[\mathrm{M}+\mathrm{Na}]^{+} 428.1910, \mathrm{~m} / z$ found 428.1904 .

\section{Coupling azido derivatives and a propargyl derivative of fenpiclonil by " click chemistry » (Fig. 1 ; compounds 11R 1-3)}

For the second step we prepared a key intermediate of fenpiclonil, substituted with an alkyne function, allowing to consider thereafter a cycloaddition-1,3 by « click chemistry » giving the 1,2,3 -triazole ring as spacer. In order to achieve this, we were interested in preparing compound $\mathbf{1 0}$ as key intermediate for synthesizing target compounds. Compound $\mathbf{9}$, previously described (Chollet et al. 2005), reacted with propargylamine in the presence of DMAP and using EDCl as condensing agent. The phenylpyrrole with an alkyne function $\mathbf{1 0}$ is obtained with $65 \%$ yield. The « click chemistry » reaction can be then considered between the alkyne $\mathbf{1 0}$ and the three azido derivatives prepared throughout the first step (compounds 2, 6, 8). The 1,3-dipolar cycloaddition reaction is catalyzed by the active $\mathrm{Cu}(\mathrm{I})$, generated from $\mathrm{Cu}$ (II) salts using sodium ascorbate as the reducing agent in a mixture tert-butanol-water. In these conditions the copper-catalyzed reaction allowed the synthesis of the 1,4-disubstituted regioisomers 11 specifically. We obtained the gluco-conjugate $\mathbf{1 1 R}_{\mathbf{1}}$ with $85 \%$ yield. The Lglutamic acid derivative $\mathbf{1 1} \mathbf{R}_{\mathbf{2}}$ and D-glutamic acid derivative $\mathbf{1 1} \mathbf{R}_{\mathbf{3}}$ were isolated with $73 \%$ and $85 \%$ yields respectively. We thought that this methodology could be extended to the synthesis of various amino acid derivatives, thus providing diversity for structure-activity relationship study.

-(2-(3-Cyano-4-(2,3-dichlorophenyl)-1H-pyrrol-1-yl)-N-(prop-2-ynyl)propanamide (compound 10).

To a solution of compound 9 (10.8 g, $35.0 \mathrm{mmol}, 1$ equiv) in anhydrous dichloromethane $(100 \mathrm{~mL})$ cooled to $0{ }^{\circ} \mathrm{C}$ was added propargylamine (4.47 mL, $70.0 \mathrm{mmol}, 2$ equiv), 1-(3- 
dimethylaminopropyl)-3-ethylcarbodiimide hydrochloride (14.1 g, $73.4 \mathrm{mmol}, 2.1$ equiv) and 4-dimethylaminopyridine ( $427 \mathrm{mg}, 3.50 \mathrm{mmol}, 0.1$ equiv). The reaction mixture was purged with nitrogen through the septum and then stirred at $0{ }^{\circ} \mathrm{C}$ for $2 \mathrm{~h}$. The mixture was allowed to reach room temperature and then stirred for $18 \mathrm{~h}$. Water was added and the organic layer was extracted twice with dichloromethane. The combined organic layers were washed with water, dried over $\mathrm{MgSO}_{4}$, filtered and concentrated under vacuum. The crude product was purified by silica gel column chromatography using pentane/ethyl acetate (6:4) as eluent to afford compound 10 as a white powder ( $7.9 \mathrm{~g}, 65 \%$ yield).

$\mathrm{Rf}=0.44$ (pentane/ethyl acetate: $6 / 4) ; \mathrm{Mp}=142-143{ }^{\circ} \mathrm{C} .{ }^{1} \mathrm{H}$ NMR $\left(400 \mathrm{MHz}\right.$, DMSO- $\left.d_{6}\right): \delta$ $8.77\left(\mathrm{t}, 1 \mathrm{H},{ }^{3} \mathrm{~J}=5.4 \mathrm{~Hz}, \mathrm{NH}\right), 7.87\left(\mathrm{~d}, 1 \mathrm{H},{ }^{4} \mathrm{~J}=2.3 \mathrm{~Hz}, \mathrm{H}_{2}\right), 7.68\left(\mathrm{dd}, 1 \mathrm{H},{ }^{3} J=7.1 \mathrm{~Hz},{ }^{4} J=2.5\right.$ $\left.\mathrm{Hz}, \mathrm{H}_{8}\right), 7.49-7.43\left(\mathrm{~m}, 2 \mathrm{H}, \mathrm{H}_{6}\right.$ and $\left.\mathrm{H}_{7}\right), 7.30\left(\mathrm{~d}, 1 \mathrm{H},{ }^{4} \mathrm{~J}=2.3 \mathrm{~Hz}, \mathrm{H}_{5}\right), 4.96\left(\mathrm{q}, 1 \mathrm{H},{ }^{3} J=7.1 \mathrm{~Hz}\right.$, $\left.\mathrm{H}_{\mathrm{b}}\right), 3.94\left(\mathrm{dd}, 2 \mathrm{H},{ }^{3} J=5.4 \mathrm{~Hz},{ }^{4} J=2.5 \mathrm{~Hz}, \mathrm{H}_{\mathrm{c}}\right), 3.22\left(\mathrm{t}, 1 \mathrm{H},{ }^{4} J=2.5 \mathrm{~Hz}, \mathrm{H}_{\mathrm{d}}\right), 1.64\left(\mathrm{~d}, 3 \mathrm{H},{ }^{3} J=\right.$ $\left.7.1 \mathrm{~Hz}, \mathrm{H}_{\mathrm{a}}\right) .{ }^{13} \mathrm{C}$ NMR $\left(100 \mathrm{MHz}, \mathrm{DMSO}-d_{6}\right): \delta 168.89(\mathrm{C}=\mathrm{O}), 133.94(\mathrm{C}-\mathrm{Cl}), 132.47(\mathrm{C}-\mathrm{Cl})$, $130.24\left(\mathrm{C}_{7}\right.$ and $\left.\mathrm{C}\right), 129.73\left(\mathrm{C}_{8}\right), 129.03\left(\mathrm{C}_{2}\right), 128.22\left(\mathrm{C}_{6}\right), 122.18\left(\mathrm{C}_{4}\right), 122.09\left(\mathrm{C}_{5}\right), 116.01$ $(\mathrm{CN}), 92.04\left(\mathrm{C}_{3}\right), 80.49(\mathrm{C} \quad), 73.57\left(\mathrm{C}_{\mathrm{d}}\right), 57.47\left(\mathrm{C}_{\mathrm{b}}\right), 28.29\left(\mathrm{C}_{\mathrm{c}}\right), 18.14\left(\mathrm{C}_{\mathrm{a}}\right)$. HRMS (ESI, $\left.\mathrm{CH}_{3} \mathrm{CN}\right): m / z$ calcd for $\mathrm{C}_{17} \mathrm{H}_{13} \mathrm{Cl}_{2} \mathrm{~N}_{3} \mathrm{O}[\mathrm{M}+\mathrm{Na}]^{+} 368.0333, m / z$ found 368.0328 .

- Representative coupling procedure for the synthesis of derivatives of fenpiclonil (compounds $\left.11 R_{1}-11 R_{3}\right)$

To a solution of compound 10 (3.47 g, $10.0 \mathrm{mmol}, 1$ equiv) in tert-butanol (28 mL) was added compound $\mathbf{N}_{3}-\mathbf{R x}$ (3.74 g, $10.0 \mathrm{mmol}, 1$ equiv). Then a solution of copper (II) sulfate pentahydrate (500 mg, $2.0 \mathrm{mmol}, 0.2$ equiv) and $L$-ascorbic acid sodium salt (794 mg, 4.0 mmol, 0.4 equiv) in water $(28 \mathrm{~mL})$ was added to the reaction mixture. The resulting solution was heated at $50{ }^{\circ} \mathrm{C}$ for $2 \mathrm{~h} 30$. After cooling to room temperature, the resulting mixture was diluted with dichloromethane. The organic layer was extracted, washed with saturated ammonium chloride solution, brine, dried over $\mathrm{MgSO}_{4}$, filtered and concentrated under vacuum. The crude product was purified by silica gel column chromatography using ethyl acetate as eluent to afford compounds $\mathbf{1 1} \mathbf{R}_{\mathbf{1}} \mathbf{- 1 1} \mathbf{R}_{\mathbf{3}}$.

- 2-(4-((2-(3-Cyano-4-(2,3-dichlorophenyl)-1H-pyrrol-1-yl)propanamido)methyl)-1H-1,2,3triazol-1-yl)-6-(ethanoyloxymethyl)tetrahydro-2H-pyran-3,4,5-triyl triethanoate (compound $\left.11 R_{1}\right)$. 
Compound was obtained following the representative procedure, using compound $\mathbf{1 0}(3.47 \mathrm{~g}$, $10.0 \mathrm{mmol}, 1$ equiv), compound 2 (3.74 g, $10.0 \mathrm{mmol}, 1$ equiv), copper (II) sulfate pentahydrate (500 mg, $2.0 \mathrm{mmol}, 0.2$ equiv), $L$-ascorbic acid sodium salt (794 mg, $4.0 \mathrm{mmol}$, 0.4 equiv). The crude product was purified by silica gel column chromatography using ethyl acetate as eluent to afford compound $\mathbf{1 1} \mathbf{R}_{1}$ as a white powder $(6.1 \mathrm{~g}, 85 \%$ yield $)$. $\mathrm{Rf}=0.62$ (ethyl acetate); $\mathrm{Mp}=107-108^{\circ} \mathrm{C} .{ }^{1} \mathrm{H}$ NMR $\left(400 \mathrm{MHz}, \mathrm{DMSO}-d_{6}\right): \delta 8.86\left(\mathrm{t}, 1 \mathrm{H},{ }^{3} J\right.$ $=5.6 \mathrm{~Hz}, \mathrm{NH}), 8.28\left(\mathrm{~s}, 1 \mathrm{H}, \mathrm{H}_{\mathrm{d}}\right), 7.87\left(\mathrm{~d}, 1 \mathrm{H},{ }^{4} \mathrm{~J}=2.2 \mathrm{~Hz}, \mathrm{H}_{2}\right), 7.68\left(\mathrm{dd}, 1 \mathrm{H},{ }^{3} J=7.1 \mathrm{~Hz},{ }^{4} J=\right.$ $\left.2.6 \mathrm{~Hz}, \mathrm{H}_{8}\right), 7.49-7.44\left(\mathrm{~m}, 2 \mathrm{H}, \mathrm{H}_{6}\right.$ and $\left.\mathrm{H}_{7}\right), 7.30\left(\mathrm{~d}, 1 \mathrm{H},{ }^{4} \mathrm{~J}=2.2 \mathrm{~Hz}, \mathrm{H}_{5}\right), 6.37\left(\mathrm{~d}, 1 \mathrm{H},{ }^{3} J=9.5\right.$ $\left.\mathrm{Hz}, \mathrm{H}_{\mathrm{e}}\right), 5.69\left(\mathrm{t}, 1 \mathrm{H},{ }^{3} J={ }^{3} J^{\prime}=9.5 \mathrm{~Hz}, \mathrm{H}_{\mathrm{f}}\right), 5.59\left(\mathrm{t}, 1 \mathrm{H},{ }^{3} J={ }^{3} J J^{\prime}=9.5 \mathrm{~Hz}, \mathrm{H}_{\mathrm{g}}\right), 5.22\left(\mathrm{t}, 1 \mathrm{H},{ }^{3} J=\right.$ $\left.{ }^{3} J^{\prime}=9.5 \mathrm{~Hz}, \mathrm{H}_{\mathrm{h}}\right), 4.98\left(\mathrm{q}, 1 \mathrm{H},{ }^{3} J=7.1 \mathrm{~Hz}, \mathrm{H}_{\mathrm{b}}\right), 4.46-4.33\left(\mathrm{~m}, 3 \mathrm{H}, \mathrm{H}_{\mathrm{c}}\right.$ and $\left.\mathrm{H}_{\mathrm{i}}\right), 4.19-4.09(\mathrm{~m}$, $\left.2 \mathrm{H}, \mathrm{H}_{\mathrm{j}}\right), 2.07,2.03,2.00,1.82\left(4 \mathrm{~s}, 12 \mathrm{H}, \mathrm{H}_{\mathrm{k}}\right), 1.67\left(\mathrm{~d}, 3 \mathrm{H},{ }^{3} \mathrm{~J}=7.1 \mathrm{~Hz}, \mathrm{H}_{\mathrm{a}}\right) .{ }^{13} \mathrm{C}$ NMR $(100$ MHz, DMSO- $\left.d_{6}\right): \delta 170.00(\mathrm{C}=\mathrm{O}), 169.56(\mathrm{C}=\mathrm{O}), 169.35(\mathrm{C}=\mathrm{O}), 169.16(\mathrm{C}=\mathrm{O}), 168.47$ $(\mathrm{C}=\mathrm{O}), 144.95(\mathrm{C}), 133.97(\mathrm{C}-\mathrm{Cl}), 132.48(\mathrm{C}-\mathrm{Cl}), 130.25\left(\mathrm{C}_{7}\right.$ and $\left.\mathrm{C}\right), 129.71\left(\mathrm{C}_{8}\right), 129.04$ $\left(\mathrm{C}_{2}\right), 128.20\left(\mathrm{C}_{6}\right), 122.18\left(\mathrm{C}_{4}\right), 122.11\left(\mathrm{C}_{5}\right), 121.95\left(\mathrm{C}_{\mathrm{d}}\right), 116.05(\mathrm{CN}), 92.00\left(\mathrm{C}_{3}\right), 83.77\left(\mathrm{C}_{\mathrm{e}}\right)$, $73.26\left(\mathrm{C}_{\mathrm{i}}\right), 72.19\left(\mathrm{C}_{\mathrm{g}}\right), 70.05\left(\mathrm{C}_{\mathrm{f}}\right), 67.50\left(\mathrm{C}_{\mathrm{h}}\right), 61.77\left(\mathrm{C}_{\mathrm{j}}\right), 57.59\left(\mathrm{C}_{\mathrm{b}}\right), 34.30\left(\mathrm{C}_{\mathrm{c}}\right)$, 20.50, 20.38, 20.24, $19.89\left(4 \mathrm{C}_{\mathrm{k}}\right), 18.29\left(\mathrm{C}_{\mathrm{a}}\right)$. HRMS (ESI, $\left.\mathrm{CH}_{3} \mathrm{CN}\right): m / z$ calcd for $\mathrm{C}_{31} \mathrm{H}_{32} \mathrm{Cl}_{2} \mathrm{~N}_{6} \mathrm{O}_{10}[\mathrm{M}+$ $\mathrm{Na}]^{+} 741.1455, \mathrm{~m} / \mathrm{z}$ found 741.1449 .

- Tert-butyl 2-(tert-butoxycarbonylamino)-5-(2-(4-((2-(3-cyano-4-(2,3-dichlorophenyl)-1Hpyrrol-1-yl)propanamido)methyl)-1H-1,2,3-triazol-1-yl)ethylamino)-5-oxopentanoate (compound 11R $\boldsymbol{R}_{2}$.

Compound was obtained following the representative procedure, using compound 10 (3.47 g, $10.0 \mathrm{mmol}, 1$ equiv), compound 6 (3.72 g, $10.0 \mathrm{mmol}, 1$ equiv), copper (II) sulfate pentahydrate (500 mg, $2.0 \mathrm{mmol}, 0.2$ equiv), $L$-ascorbic acid sodium salt (794 mg, $4.0 \mathrm{mmol}$, 0.4 equiv). The crude product was purified by silica gel column chromatography using ethyl acetate as eluent to afford compound $\mathbf{1 1 R _ { 2 }}$ as a white powder (5.3 g, 73\% yield). $\mathrm{Rf}=0.59$ (ethyl acetate/methanol: 9/1); Mp = 117-118 ${ }^{\circ} \mathrm{C} .{ }^{1} \mathrm{H}$ NMR $\left(400 \mathrm{MHz}, \mathrm{DMSO}-d_{6}\right): \delta$ $8.82\left(\mathrm{t}, 1 \mathrm{H},{ }^{3} J=5.1 \mathrm{~Hz}, \mathrm{NH}\right), 8.04\left(\mathrm{t}, 1 \mathrm{H},{ }^{3} J=5.6 \mathrm{~Hz}, \mathrm{NH}\right), 7.97\left(\mathrm{~s}, 1 \mathrm{H}, \mathrm{H}_{\mathrm{d}}\right), 7.87\left(\mathrm{~d}, 1 \mathrm{H},{ }^{4} J=\right.$ $\left.2.2 \mathrm{~Hz}, \mathrm{H}_{2}\right), 7.68\left(\mathrm{dd}, 1 \mathrm{H},{ }^{3} \mathrm{~J}=7.2 \mathrm{~Hz},{ }^{4} \mathrm{~J}=2.4 \mathrm{~Hz}, \mathrm{H}_{8}\right), 7.48-7.42\left(\mathrm{~m}, 2 \mathrm{H}, \mathrm{H}_{6}\right.$ and $\left.\mathrm{H}_{7}\right), 7.30$ $\left(\mathrm{d}, 1 \mathrm{H},{ }^{4} J=2.2 \mathrm{~Hz}, \mathrm{H}_{5}\right), 7.15\left(\mathrm{~d}, 1 \mathrm{H},{ }^{3} J=7.7 \mathrm{~Hz}, \mathrm{NH}\right), 4.98\left(\mathrm{q}, 1 \mathrm{H},{ }^{3} J=7.0 \mathrm{~Hz}, \mathrm{H}_{\mathrm{b}}\right), 4.43-$ $4.32\left(\mathrm{~m}, 4 \mathrm{H}, \mathrm{H}_{\mathrm{e}}\right.$ and $\left.\mathrm{H}_{\mathrm{f}}\right), 3.83-3.77\left(\mathrm{~m}, 1 \mathrm{H}, \mathrm{H}_{\mathrm{i}}\right), 3.52-3.47\left(\mathrm{~m}, 2 \mathrm{H}, \mathrm{H}_{\mathrm{c}}\right), 2.15\left(\mathrm{t}, 2 \mathrm{H},{ }^{3} J=7.6\right.$ $\left.\mathrm{Hz}, \mathrm{H}_{\mathrm{g}}\right), 1.95-1.70\left(\mathrm{~m}, 2 \mathrm{H}, \mathrm{H}_{\mathrm{h}}\right), 1.65\left(\mathrm{~d}, 3 \mathrm{H},{ }^{3} \mathrm{~J}=7.0 \mathrm{~Hz}, \mathrm{H}_{\mathrm{a}}\right), 1.44-1.42\left(\mathrm{~m}, 18 \mathrm{H}, \mathrm{H}_{\mathrm{j}}\right.$ and $\left.\mathrm{H}_{\mathrm{k}}\right)$. ${ }^{13} \mathrm{C}$ NMR (100 MHz, DMSO- $\left.d_{6}\right): \delta 171.74(\mathrm{C}=\mathrm{O}), 171.54(\mathrm{C}=\mathrm{O}), 169.02(\mathrm{C}=\mathrm{O}), 155.48$ 
$(\mathrm{C}=\mathrm{O}), 143.97(\mathrm{C}), 133.95(\mathrm{C}-\mathrm{Cl}), 132.47(\mathrm{C}-\mathrm{Cl}), 130.23\left(\mathrm{C}_{7}\right.$ and $\left.\mathrm{C}\right), 129.70\left(\mathrm{C}_{8}\right), 128.98$ $\left(\mathrm{C}_{2}\right), 128.19\left(\mathrm{C}_{6}\right), 123.18\left(\mathrm{C}_{\mathrm{d}}\right), 122.16\left(\mathrm{C}_{4}\right), 122.05\left(\mathrm{C}_{5}\right), 116.02(\mathrm{CN}), 92.00\left(\mathrm{C}_{3}\right), 80.27(\mathrm{C})$, $78.04(\mathrm{C}), 57.57\left(\mathrm{C}_{\mathrm{b}}\right), 53.93\left(\mathrm{C}_{\mathrm{i}}\right), 48.66\left(\mathrm{C}_{\mathrm{e}}\right), 38.89\left(\mathrm{C}_{\mathrm{c}}\right), 34.45\left(\mathrm{C}_{\mathrm{f}}\right), 31.56\left(\mathrm{C}_{\mathrm{g}}\right), 28.16\left(3 \mathrm{C}_{\mathrm{k}}\right)$, $27.61\left(3 \mathrm{C}_{\mathrm{j}}\right), 26.44\left(\mathrm{C}_{\mathrm{h}}\right), 18.27\left(\mathrm{C}_{\mathrm{a}}\right)$. HRMS (ESI, $\left.\mathrm{CH}_{3} \mathrm{CN}\right): \mathrm{m} / z$ calcd for $\mathrm{C}_{33} \mathrm{H}_{42} \mathrm{Cl}_{2} \mathrm{~N}_{8} \mathrm{O}_{6}[\mathrm{M}+$ $\mathrm{Na}]^{+} 739.2502, \mathrm{~m} / \mathrm{z}$ found 739.2497 .

- Benzyl 2-(tert-butoxycarbonylamino)-5-(2-(4-((2-(3-cyano-4-(2,3-dichlorophenyl)-1Hpyrrol-1-yl)propanamido)methyl)-1H-1,2,3-triazol-1-yl)ethylamino)-5-oxopentanoate (compound 11R $\mathbf{R}_{3}$.

Compound was obtained following the representative procedure, using compound 10 (3.47 $\mathrm{g}$, 10.0 mmol, 1 equiv), compound 8 (4.06 g, 10.0 mmol, 1 equiv), copper (II) sulfate pentahydrate (500 mg, $2.0 \mathrm{mmol}, 0.2$ equiv), $L$-ascorbic acid sodium salt (794 mg, $4.0 \mathrm{mmol}$, 0.4 equiv). The crude product was purified by silica gel column chromatography using ethyl acetate as eluent to afford compound $\mathbf{1 1 R _ { 3 }}$ as a white powder $(6.4 \mathrm{~g}, 85 \%$ yield). $\mathrm{Rf}=0.18$ (ethyl acetate); $\mathrm{Mp}=156-157^{\circ} \mathrm{C} .{ }^{1} \mathrm{H}$ NMR $\left(400 \mathrm{MHz}, \mathrm{DMSO}-d_{6}\right): \delta 8.80\left(\mathrm{t}, 1 \mathrm{H},{ }^{3} J\right.$ $=5.4 \mathrm{~Hz}, \mathrm{NH}), 8.09\left(\mathrm{t}, 1 \mathrm{H},{ }^{3} J=5.7 \mathrm{~Hz}, \mathrm{NH}\right), 7.96\left(\mathrm{~s}, 1 \mathrm{H}, \mathrm{H}_{\mathrm{d}}\right), 7.87\left(\mathrm{~d}, 1 \mathrm{H},{ }^{4} J=2.3 \mathrm{~Hz}, \mathrm{H}_{2}\right)$, $7.68\left(\mathrm{dd}, 1 \mathrm{H},{ }^{3} \mathrm{~J}=7.2 \mathrm{~Hz},{ }^{4} \mathrm{~J}=2.4 \mathrm{~Hz}, \mathrm{H}_{8}\right), 7.48-7.42\left(\mathrm{~m}, 2 \mathrm{H}, \mathrm{H}_{6}\right.$ and $\left.\mathrm{H}_{7}\right), 7.40-7.33(\mathrm{~m}, 5 \mathrm{H}$, $\mathrm{H}_{\mathrm{l}}, \mathrm{H}_{\mathrm{m}}$ and $\left.\mathrm{H}_{\mathrm{n}}\right), 7.30\left(\mathrm{~d}, 1 \mathrm{H},{ }^{4} \mathrm{~J}=2.3 \mathrm{~Hz}, \mathrm{H}_{5}\right), 7.00\left(\mathrm{~d}, 1 \mathrm{H},{ }^{3} \mathrm{~J}=8.0 \mathrm{~Hz}, \mathrm{NH}\right), 5.12\left(\mathrm{~s}, 2 \mathrm{H}, \mathrm{H}_{\mathrm{k}}\right)$, $4.98\left(\mathrm{q}, 1 \mathrm{H},{ }^{3} J=7.0 \mathrm{~Hz}, \mathrm{H}_{\mathrm{b}}\right), 4.43\left(\mathrm{t}, 2 \mathrm{H},{ }^{3} J=6.1 \mathrm{~Hz}, \mathrm{H}_{\mathrm{e}}\right), 4.38-4.34$ (m, 2H, H $\left.\mathrm{H}_{\mathrm{f}}\right), 3.95-3.89$ $\left(\mathrm{m}, 1 \mathrm{H}, \mathrm{H}_{\mathrm{i}}\right), 3.60-3.49\left(\mathrm{~m}, 2 \mathrm{H}, \mathrm{H}_{\mathrm{c}}\right), 2.37\left(\mathrm{t}, 2 \mathrm{H},{ }^{3} \mathrm{~J}=7.1 \mathrm{~Hz}, \mathrm{H}_{\mathrm{g}}\right), 1.95-1.71\left(\mathrm{~m}, 2 \mathrm{H}, \mathrm{H}_{\mathrm{h}}\right), 1.65$ $\left(\mathrm{d}, 3 \mathrm{H},{ }^{3} J=7.0 \mathrm{~Hz}, \mathrm{H}_{\mathrm{a}}\right), 1.40\left(\mathrm{~s}, 9 \mathrm{H}, \mathrm{H}_{\mathrm{j}}\right) .{ }^{13} \mathrm{C} \mathrm{NMR}\left(100 \mathrm{MHz}, \mathrm{DMSO}-d_{6}\right): \delta 172.21(\mathrm{C}=\mathrm{O})$, $171.94(\mathrm{C}=\mathrm{O}), 169.00(\mathrm{C}=\mathrm{O}), 155.34(\mathrm{C}=\mathrm{O}), 143.83(\mathrm{C}), 136.18(\mathrm{C}), 133.96(\mathrm{C}-\mathrm{Cl}), 132.49$ $(\mathrm{C}-\mathrm{Cl}), 130.24\left(\mathrm{C}_{7}\right.$ and $\left.\mathrm{C}\right), 129.71\left(\mathrm{C}_{8}\right), 129.01\left(\mathrm{C}_{2}\right), 128.40\left(2 \mathrm{C}_{\mathrm{m}}\right), 128.21\left(\mathrm{C}_{6}\right), 127.95\left(\mathrm{C}_{\mathrm{n}}\right)$, $127.85\left(2 \mathrm{C}_{1}\right), 123.25\left(\mathrm{C}_{\mathrm{d}}\right), 122.17\left(\mathrm{C}_{4}\right), 122.07\left(\mathrm{C}_{5}\right), 116.04(\mathrm{CN}), 92.00\left(\mathrm{C}_{3}\right), 78.18(\mathrm{C})$, $65.45\left(\mathrm{C}_{\mathrm{k}}\right), 57.57\left(\mathrm{C}_{\mathrm{b}}\right), 53.51\left(\mathrm{C}_{\mathrm{i}}\right), 48.54\left(\mathrm{C}_{\mathrm{e}}\right), 38.89\left(\mathrm{C}_{\mathrm{c}}\right), 34.43\left(\mathrm{C}_{\mathrm{f}}\right), 30.04\left(\mathrm{C}_{\mathrm{g}}\right), 28.15\left(3 \mathrm{C}_{\mathrm{j}}\right)$, $26.97\left(\mathrm{C}_{\mathrm{h}}\right), 18.28\left(\mathrm{C}_{\mathrm{a}}\right)$. HRMS (ESI, $\left.\mathrm{CH}_{3} \mathrm{CN}\right): \mathrm{m} / z$ calcd for $\mathrm{C}_{36} \mathrm{H}_{40} \mathrm{Cl}_{2} \mathrm{~N}_{8} \mathrm{O}_{6}[\mathrm{M}+\mathrm{Na}]^{+}$ $773.2346, \mathrm{~m} / \mathrm{z}$ found 773.2340 .

\section{Deprotection of the sugar or amino acid moiety (Fig. 1 ; compounds 12, L-13, D-13)}

The third step consisted of the selective deprotection of the sugar or amino acid moiety of the compounds $\mathbf{1 1} \mathbf{R}_{\mathbf{1}}, \mathbf{1 1} \mathbf{R}_{\mathbf{2}}$ and $\mathbf{1 1} \mathbf{R}_{\mathbf{3}}$. The protecting acetyl groups of the hydroxyl functions of the $\beta$-D-glucose of $\mathbf{1 1} \mathbf{R}_{\mathbf{1}}$ were O-deacetylated by treatment with sodium methoxide, prepared in situ. Then the obtained alkoxide was neutralized by an acid cation exchange resin 
(Amberlyst IRN 77) giving the final compound 12 with $60 \%$ yield. The deprotection of the alpha-aminoacid function of $\mathbf{1 1} \mathbf{R}_{\mathbf{2}}$ was achieved by trifluoroacetic acid in dichloromethane for $7 \mathrm{~h}$ at room temperature, afforded the final conjugate $\mathbf{L - 1 3}$ with 98\% yield (Siebum et al. 2004). The two protecting groups of the alpha-aminoacid function of $\mathbf{1 1} \mathbf{R}_{\mathbf{3}}$ being different, it is necessary to proceed in two steps. First the benzyl group protecting the carboxylic acid function is hydrogenated in presence of palladium on carbon in methanol for $1 \mathrm{~h} 30$ at room temperature (Usuki et al. 2014). This compound is thus treated with trifluoroacetic acid in dichloromethane for $1 \mathrm{~h}$ at room temperature, permitting to remove the protecting $t$ butoxycarbonyl group of the amino function and led to the final compound D-13 with $98 \%$ yield.

-2-(3-Cyano-4-(2,3-dichlorophenyl)-1H-pyrrol-1-yl)-N-((1-(3,4,5-trihydroxy-6(hydroxymethyl)tetrahydro-2H-pyran-2-yl)-1H-1,2,3-triazol-4-yl)methyl)propanamide (compound 12).

To a solution of the compound $\mathbf{1 1 R}_{\mathbf{1}}(2.20 \mathrm{~g}, 3.0 \mathrm{mmol}, 1$ equiv) in methanol (12 $\mathrm{mL})$ was added sodium (169 mg, $7.3 \mathrm{mmol}, 2.4$ equiv). The reaction mixture was purged with nitrogen through the septum and then stirred at room temperature for 15 minutes. The reaction mixture was neutralized with Amberlyst 77. The insoluble materials were separated by filtration through celite eluting with methanol. The filtrate was then concentrated under vacuum to afford the compound 12 as a white powder (1.0 g, 60\% yield).

$\mathrm{Rf}=0.12$ (ethyl acetate/methanol: 9/1); $\mathrm{Mp}=138-139{ }^{\circ} \mathrm{C} ;[\alpha]^{20} \mathrm{D}-3.6\left(\mathrm{c}=0.4\right.$, acetone). ${ }^{1} \mathrm{H}$ NMR (400 MHz, DMSO- $\left.d_{6}\right): \delta 8.86\left(\mathrm{t}, 1 \mathrm{H},{ }^{3} J=5.5 \mathrm{~Hz}, \mathrm{NH}\right), 8.17\left(\mathrm{~s}, 1 \mathrm{H}, \mathrm{H}_{\mathrm{d}}\right), 7.89\left(\mathrm{~d}, 1 \mathrm{H},{ }^{4} J\right.$ $\left.=2.2 \mathrm{~Hz}, \mathrm{H}_{2}\right), 7.68\left(\mathrm{dd}, 1 \mathrm{H},{ }^{3} J=7.1 \mathrm{~Hz},{ }^{4} J=2.5 \mathrm{~Hz}, \mathrm{H}_{8}\right), 7.49-7.43\left(\mathrm{~m}, 2 \mathrm{H}, \mathrm{H}_{6}\right.$ and $\left.\mathrm{H}_{7}\right), 7.32$ $\left(\mathrm{d}, 1 \mathrm{H},{ }^{4} J=2.2 \mathrm{~Hz}, \mathrm{H}_{5}\right), 5.56\left(\mathrm{~d}, 1 \mathrm{H},{ }^{3} J=9.3 \mathrm{~Hz}, \mathrm{H}_{\mathrm{e}}\right), 5.41\left(\mathrm{~d}, 1 \mathrm{H},{ }^{3} J=6.0 \mathrm{~Hz}, \mathrm{OH}\right), 5.34(\mathrm{~d}$, $\left.1 \mathrm{H},{ }^{3} J=5.0 \mathrm{~Hz}, \mathrm{OH}\right), 5.21\left(\mathrm{~d}, 1 \mathrm{H},{ }^{3} J=5.5 \mathrm{~Hz}, \mathrm{OH}\right), 4.99\left(\mathrm{q}, 1 \mathrm{H},{ }^{3} J=7.0 \mathrm{~Hz}, \mathrm{H}_{\mathrm{b}}\right), 4.68$ (t, $\left.1 \mathrm{H},{ }^{3} J=5.5 \mathrm{~Hz}, \mathrm{OH}\right), 4.47-4.35\left(\mathrm{~m}, 2 \mathrm{H}, \mathrm{H}_{\mathrm{c}}\right), 3.77-3.71\left(\mathrm{~m}, 2 \mathrm{H}, \mathrm{H}_{\mathrm{f}}\right.$ and $\left.\mathrm{H}_{\mathrm{j}}\right), 3.48-3.43(\mathrm{~m}, 3 \mathrm{H}$, $\mathrm{H}_{\mathrm{g}}, \mathrm{H}_{\mathrm{i}}$ and $\left.\mathrm{H}_{\mathrm{j}}\right), 3.29-3.23\left(\mathrm{~m}, 1 \mathrm{H}, \mathrm{H}_{\mathrm{h}}\right), 1.67\left(\mathrm{~d}, 3 \mathrm{H},{ }^{3} \mathrm{~J}=7.0 \mathrm{~Hz}, \mathrm{H}_{\mathrm{a}}\right) .{ }^{13} \mathrm{C} \mathrm{NMR}(100 \mathrm{MHz}$, DMSO- $\left.d_{6}\right)$ : $\delta 169.16(\mathrm{C}=\mathrm{O}), 144.02(\mathrm{C}), 133.99(\mathrm{C}-\mathrm{Cl}), 132.49(\mathrm{C}-\mathrm{Cl}), 130.30\left(\mathrm{C}_{7}\right), 130.27$ $(\mathrm{C}), 129.74\left(\mathrm{C}_{8}\right), 129.09\left(\mathrm{C}_{2}\right), 128.26\left(\mathrm{C}_{6}\right), 122.20\left(\mathrm{C}_{5}\right), 122.14\left(\mathrm{C}_{4}\right.$ and $\left.\mathrm{C}_{\mathrm{d}}\right), 116.09(\mathrm{CN})$, $92.02\left(\mathrm{C}_{3}\right), 87.43\left(\mathrm{C}_{\mathrm{e}}\right), 79.98\left(\mathrm{C}_{\mathrm{i}}\right), 77.03\left(\mathrm{C}_{\mathrm{g}}\right), 72.03\left(\mathrm{C}_{\mathrm{f}}\right), 69.61\left(\mathrm{C}_{\mathrm{h}}\right), 60.78\left(\mathrm{C}_{\mathrm{j}}\right), 57.61\left(\mathrm{C}_{\mathrm{b}}\right)$, $34.48\left(\mathrm{C}_{\mathrm{c}}\right), 18.33\left(\mathrm{C}_{\mathrm{a}}\right)$. HRMS (ESI, $\left.\mathrm{CH}_{3} \mathrm{CN}\right): \mathrm{m} / z$ calcd for $\mathrm{C}_{23} \mathrm{H}_{24} \mathrm{Cl}_{2} \mathrm{~N}_{6} \mathrm{O}_{6}[\mathrm{M}+\mathrm{H}]^{+}$ $551.1213, \mathrm{~m} / \mathrm{z}$ found 551.1207 . 
-2-Amino-5-(2-(4-((2-(3-cyano-4-(2,3-dichlorophenyl)-1H-pyrrol-1-yl)propanamido)methyl)1H-1,2,3-triazol-1-yl)ethylamino)-5-oxopentanoic acid (compound L-13).

The compound $\mathbf{1 1 R}_{\mathbf{2}}(2.15 \mathrm{~g}, 3.0 \mathrm{mmol}, 1$ equiv) was diluted in a mixture anhydrous dichloromethane - trifluoroacetic acid $(24 \mathrm{~mL}, 1: 1)$. The reaction mixture was purged with nitrogen through the septum and then stirred at room temperature for $7 \mathrm{~h}$ before being evaporated. The residue was co-evaporated with ethyl acetate, dried under vacuum to afford the compound L-13 as a beige powder (1.6 g, 98\% yield). $\mathrm{Rf}=0.12$ (ethyl acetate/methanol: $5 / 5) ; \mathrm{Mp}=137-138^{\circ} \mathrm{C} ;[\alpha]^{20} \mathrm{D}-3.2\left(c=1.2\right.$, acetone). ${ }^{1} \mathrm{H}$ NMR (400 MHz, DMSO- $\left.d_{6}\right): \delta 8.87\left(\mathrm{t}, 1 \mathrm{H},{ }^{3} J=5.5 \mathrm{~Hz}, \mathrm{NH}\right), 8.33\left(\mathrm{~s}, 3 \mathrm{H}, \mathrm{NH}_{2}\right.$ and $\left.\mathrm{OH}\right), 8.21$ (t, $\left.1 \mathrm{H},{ }^{3} J=5.6 \mathrm{~Hz}, \mathrm{NH}\right), 7.98\left(\mathrm{~s}, 1 \mathrm{H}, \mathrm{H}_{\mathrm{d}}\right), 7.87\left(\mathrm{~d}, 1 \mathrm{H},{ }^{4} \mathrm{~J}=2.3 \mathrm{~Hz}, \mathrm{H}_{2}\right), 7.68\left(\mathrm{dd}, 1 \mathrm{H},{ }^{3} J=7.4\right.$ $\left.\mathrm{Hz},{ }^{4} J=2.2 \mathrm{~Hz}, \mathrm{H}_{8}\right), 7.49-7.42\left(\mathrm{~m}, 2 \mathrm{H}, \mathrm{H}_{6}\right.$ and $\left.\mathrm{H}_{7}\right), 7.30\left(\mathrm{~d}, 1 \mathrm{H},{ }^{4} J=2.3 \mathrm{~Hz}, \mathrm{H}_{5}\right), 4.99(\mathrm{q}, 1 \mathrm{H}$, $\left.{ }^{3} J=7.1 \mathrm{~Hz}, \mathrm{H}_{\mathrm{b}}\right), 4.43\left(\mathrm{t}, 2 \mathrm{H},{ }^{3} J=6.2 \mathrm{~Hz}, \mathrm{H}_{\mathrm{e}}\right), 4.39-4.36\left(\mathrm{~m}, 2 \mathrm{H}, \mathrm{H}_{\mathrm{f}}\right), 3.96-3.94\left(\mathrm{~m}, 1 \mathrm{H}, \mathrm{H}_{\mathrm{i}}\right)$, 3.53-3.49 (m, 2H, $\left.\mathrm{H}_{\mathrm{c}}\right), 2.32-2.20\left(\mathrm{~m}, 2 \mathrm{H}, \mathrm{H}_{\mathrm{g}}\right), 1.95-1.89\left(\mathrm{~m}, 2 \mathrm{H}, \mathrm{H}_{\mathrm{h}}\right), 1.65\left(\mathrm{~d}, 3 \mathrm{H},{ }^{3} \mathrm{~J}=7.1 \mathrm{~Hz}\right.$, $\left.\mathrm{H}_{\mathrm{a}}\right) \cdot{ }^{13} \mathrm{C}$ NMR $\left(100 \mathrm{MHz}, \mathrm{DMSO}-d_{6}\right): \delta 172.02(\mathrm{C}=\mathrm{O}), 170.82(\mathrm{C}=\mathrm{O}), 169.10(\mathrm{C}=\mathrm{O}), 144.11$ (C), $133.97(\mathrm{C}-\mathrm{Cl}), 132.50(\mathrm{C}-\mathrm{Cl}), 130.26\left(\mathrm{C}_{7}\right.$ and $\left.\mathrm{C}\right), 129.74\left(\mathrm{C}_{8}\right), 129.01\left(\mathrm{C}_{2}\right), 128.23\left(\mathrm{C}_{6}\right)$, $123.17\left(\mathrm{C}_{\mathrm{d}}\right), 122.19\left(\mathrm{C}_{4}\right), 122.08\left(\mathrm{C}_{5}\right), 116.06(\mathrm{CN}), 92.01\left(\mathrm{C}_{3}\right), 57.58\left(\mathrm{C}_{\mathrm{b}}\right), 51.61\left(\mathrm{C}_{\mathrm{i}}\right), 48.63$ $\left(\mathrm{C}_{\mathrm{e}}\right), 38.89\left(\mathrm{C}_{\mathrm{c}}\right), 34.47\left(\mathrm{C}_{\mathrm{f}}\right), 30.52\left(\mathrm{C}_{\mathrm{g}}\right), 25.83\left(\mathrm{C}_{\mathrm{h}}\right), 18.28\left(\mathrm{C}_{\mathrm{a}}\right)$. HRMS (ESI, $\left.\mathrm{CH}_{3} \mathrm{CN}\right): \mathrm{m} / \mathrm{z}$ calcd for $\mathrm{C}_{24} \mathrm{H}_{26} \mathrm{Cl}_{2} \mathrm{~N}_{8} \mathrm{O}_{4}[\mathrm{M}+\mathrm{H}]^{+} 561.1532, \mathrm{~m} / z$ found 561.1527 .

-2-Amino-5-(2-(4-((2-(3-cyano-4-(2,3-dichlorophenyl)-1H-pyrrol-1-yl)propanamido)methyl)1H-1,2,3-triazol-1-yl)ethylamino)-5-oxopentanoic acid (compound D-13). 2-(Tert-butoxycarbonylamino)-5-(2-(4-((2-(3-cyano-4-(2,3-dichlorophenyl)-1H-pyrrol-1yl)propanamido)methyl)-1H-1,2,3-triazol-1-yl)ethylamino)-5-oxopentanoic acid (compound $\left.11 R_{3}{ }^{\prime}\right)$

A solution of the compound $\mathbf{1 1 R}_{\mathbf{3}}(3.76 \mathrm{~g}, 5.00 \mathrm{mmol}, 1$ equiv) in methanol (23 $\mathrm{mL})$ was treated with $\mathrm{Pd} / \mathrm{C}(10 \%, 53.2 \mathrm{mg})$ and hydrogenated under balloon pressure at room temperature. After stirring for $1 \mathrm{~h} 30$ at room temperature, the insoluble materials were separated by filtration through celite eluting with methanol. The filtrate was then concentrated under vacuum to afford the compound $\mathbf{1 1} \mathbf{R}_{\mathbf{3}}$ ' as a white powder (3.3 g, $99 \%$ yield). $\mathrm{Rf}=0.14$ (ethyl acetate/methanol: 9/1); $\mathrm{Mp}=153-154{ }^{\circ} \mathrm{C} .{ }^{1} \mathrm{H}$ NMR $\left(400 \mathrm{MHz}, \mathrm{DMSO}-d_{6}\right)$ : ठ $12.13(\mathrm{~s}, 1 \mathrm{H}, \mathrm{OH}), 8.80\left(\mathrm{t}, 1 \mathrm{H},{ }^{3} J=5.4 \mathrm{~Hz}, \mathrm{NH}\right), 8.07$ (t, $\left.1 \mathrm{H},{ }^{3} J=5.6 \mathrm{~Hz}, \mathrm{NH}\right), 7.96(\mathrm{~s}, 1 \mathrm{H}$, $\left.\mathrm{H}_{\mathrm{d}}\right), 7.87\left(\mathrm{~d}, 1 \mathrm{H},{ }^{4} \mathrm{~J}=2.2 \mathrm{~Hz}, \mathrm{H}_{2}\right), 7.68\left(\mathrm{dd}, 1 \mathrm{H},{ }^{3} J=7.2 \mathrm{~Hz},{ }^{4} \mathrm{~J}=2.4 \mathrm{~Hz}, \mathrm{H}_{8}\right), 7.48-7.42(\mathrm{~m}$, $2 \mathrm{H}, \mathrm{H}_{6}$ and $\left.\mathrm{H}_{7}\right), 7.30\left(\mathrm{~d}, 1 \mathrm{H},{ }^{4} J=2.2 \mathrm{~Hz}, \mathrm{H}_{5}\right), 6.94\left(\mathrm{~d}, 1 \mathrm{H},{ }^{3} J=7.9 \mathrm{~Hz}, \mathrm{NH}\right), 4.98\left(\mathrm{q}, 1 \mathrm{H},{ }^{3} J=\right.$ 
$\left.7.0 \mathrm{~Hz}, \mathrm{H}_{\mathrm{b}}\right), 4.43\left(\mathrm{t}, 2 \mathrm{H},{ }^{3} J=6.2 \mathrm{~Hz}, \mathrm{H}_{\mathrm{e}}\right), 4.39-4.35\left(\mathrm{~m}, 2 \mathrm{H}, \mathrm{H}_{\mathrm{f}}\right), 3.92-3.86\left(\mathrm{~m}, 1 \mathrm{H}, \mathrm{H}_{\mathrm{i}}\right), 3.61-$ $3.49\left(\mathrm{~m}, 2 \mathrm{H}, \mathrm{H}_{\mathrm{c}}\right), 2.23\left(\mathrm{t}, 2 \mathrm{H},{ }^{3} J=7.6 \mathrm{~Hz}, \mathrm{H}_{\mathrm{g}}\right), 1.86-1.64\left(\mathrm{~m}, 2 \mathrm{H}, \mathrm{H}_{\mathrm{h}}\right), 1.62\left(\mathrm{~d}, 3 \mathrm{H},{ }^{3} J=7.0\right.$ $\left.\mathrm{Hz}, \mathrm{H}_{\mathrm{a}}\right), 1.40\left(\mathrm{~s}, 9 \mathrm{H}, \mathrm{H}_{\mathrm{j}}\right) .{ }^{13} \mathrm{C} \mathrm{NMR}\left(100 \mathrm{MHz}, \mathrm{DMSO}-d_{6}\right): \delta 173.95(\mathrm{C}=\mathrm{O}), 172.13(\mathrm{C}=\mathrm{O})$, $169.03(\mathrm{C}=\mathrm{O}), 155.36(\mathrm{C}=\mathrm{O}), 143.83(\mathrm{C}), 133.98(\mathrm{C}-\mathrm{Cl}), 132.50(\mathrm{C}-\mathrm{Cl}), 130.26\left(\mathrm{C}_{7}\right.$ and $\left.\mathrm{C}\right)$, $129.73\left(\mathrm{C}_{8}\right), 129.02\left(\mathrm{C}_{2}\right), 128.23\left(\mathrm{C}_{6}\right), 123.28\left(\mathrm{C}_{\mathrm{d}}\right), 122.19\left(\mathrm{C}_{4}\right), 122.09\left(\mathrm{C}_{5}\right), 116.07(\mathrm{CN})$, $92.00\left(\mathrm{C}_{3}\right), 78.16(\mathrm{C}), 57.59\left(\mathrm{C}_{\mathrm{b}}\right), 53.69\left(\mathrm{C}_{\mathrm{i}}\right), 48.55\left(\mathrm{C}_{\mathrm{e}}\right), 38.89\left(\mathrm{C}_{\mathrm{c}}\right), 34.45\left(\mathrm{C}_{\mathrm{f}}\right), 30.15\left(\mathrm{C}_{\mathrm{g}}\right)$, $28.18\left(3 \mathrm{C}_{\mathrm{j}}\right), 27.06\left(\mathrm{C}_{\mathrm{h}}\right), 18.30\left(\mathrm{C}_{\mathrm{a}}\right)$. HRMS (ESI, $\left.\mathrm{CH}_{3} \mathrm{CN}\right): \mathrm{m} / z$ calcd for $\mathrm{C}_{29} \mathrm{H}_{34} \mathrm{Cl}_{2} \mathrm{~N}_{8} \mathrm{O}_{6}[\mathrm{M}+$ $\mathrm{Na}]^{+} 683.1876, m / z$ found 683.1871 .

The compound 11R $\mathbf{R}_{\mathbf{3}}$ ' (1.98 g, $3.0 \mathrm{mmol}, 1$ equiv) was diluted in a mixture anhydrous dichloromethane - trifluoroacetic acid $(10.2 \mathrm{~mL}, 1: 1)$. The reaction mixture was purged with nitrogen through the septum and then stirred at room temperature for $1 \mathrm{~h}$ before being evaporated. The residue was co-evaporated with ethyl acetate, dried under vacuum to afford the compound D-13 as a rose powder (1.6 g, 98\% yield).

\section{Phloem mobility of compounds $12, \mathrm{~L}-13$ and $D-13$}

\section{Comparison of phloem systemicity of compounds 12 and 13 in the Ricinus model}

Phloem systemicity of conjugate $\mathbf{1 2}$ and $\mathbf{1 3}$ was studied in the Ricinus seedlings, which was widely used to test the phloem mobility of xenobiotics (Chollet et al. 2005, Delétage-Grandon et al. 2001, Wu et al. 2012, Yang et al. 2011). One hour after the beginning of incubation of Ricinus cotyledons in the standard solution with compound $\mathbf{L - 1 3}$ at $100 \mu \mathrm{M}$ concentration, the amino acid conjugate was found in the phloem sap. Its concentration increased up to 5 hours to reach about $12 \mu \mathrm{M}$ (Fig. 3A). By contrast, the phloem systemicity of compound 12 was extremely low. Its concentration in the phloem sap was about twenty times lower than that of compound L-13 (compare Fig. 3A and Fig. 4A). Consequently, a higher concentration of compound $12(500 \mu \mathrm{M})$ in the incubation medium was required for time course experiments. Under this latter experimental condition, it could be noted that the concentration of the glucose conjugate increased up to 4 hours to reach a plateau around $3 \mu \mathrm{M}$ (Fig. 4B). Glucose is poorly present in the phloem sap of Ricinus seedlings (about $2 \mathrm{mM}$ ). However, when cotyledons were dipped in a solution containing $200 \mathrm{mM}$ glucose, the same concentration was found in the sap after two hours of incubation (Kallarackal and Komor 1989). Therefore, the extremely low systemicity of compound $\mathbf{1 2}$ at $\mathrm{pH} 5.0$ is not due to membrane and/or metabolic mechanisms preventing glucose to enter the sieve tubes as it is the case in various species which translocate only sucrose (Barlow and Randolph 1978, 
Dinant et al. 2010, Girousse et al. 1991, Hayashi and Chino 1986, Liu et al. 2012). In that respect, fipronil-glucose conjugates exhibited phloem systemicity in Ricinus seedlings (Wu et al. 2012, Yang et al. 2011).

\section{Mechanisms governing phloem mobility of compound 13}

In drug discovery, passive membrane permeability of drug candidates can be predicted using Lipinski's rule of five (Ro5) and other simplified rule established by Veber (Lipinski et al. 2012, Veber et al. 2002). The same approach was adopted to profile agrochemicals (Avram et al. 2014, Tice 2001). Coupling glucose or glutamic acid with the parent compound 9 modified many chemical descriptors and physicochemical properties (Table II). Molecular weight, hydrogen bond donors, hydrogen bond acceptors, free rotatable bonds and polar surface area values increased dramatically whereas Log D value at pH 5.0 decreased. Surprisingly, the chemical descriptors and physicochemical values of the glucose conjugate $\mathbf{1 2}$ are very similar to those of the amino acid conjugate 13. The computed physicochemical properties of 12 and 13 violate the Ro5 and Veber rule (Table II), but both conjugates were detected in phloem sap. It should be pointed out that such prediction approaches are not applicable to actively transported compounds (Rocher et al. 2009). Taking into account the extremely poor systemicity of compound $\mathbf{1 2}$, the study of transport properties of compound $\mathbf{9}$ derivatives was focused on amino acid conjugates, more especially on compound L-13. It has been known for a long time that the positional relationship between the alpha-amino and carboxyl groups is an important parameter in substrate recognition by amino acid carriers (Frommer et al. 1994, Li and Bush 1992). Therefore, the phloem systemicity of the amino acid conjugate D-13 was studied and compared to that of conjugate L-13. The dramatic difference in the ability of L-13 and D-13 (Fig. 3A and 3B) to move in the phloem suggests that a stereospecific amino acid carrier system is involved in L-13 uptake. In addition, the phloem transport of L-13 was sensitive to $\mathrm{pH}$ changes in the incubation medium (Fig 5). Under our experimental conditions, L-13 systemicity was optimal at $\mathrm{pH} 5.0$ and was reduced by about four times (amounts exported) and five times (concentration of phloem sap) at pH 7.0 and 8.0 (Fig. 5A and 5B). A similar $\mathrm{pH}$ dependence was noted in the past for neutral amino acids uptake in foliar tissues (Despeghel and Delrot 1983). By contrast, the phloem transport of acidic derivatives of fenpiclonil, similar (Chollet et al. 2004) or 3 fold higher (Chollet et al. 2005) at pH 5.0 than that of compound L-13, decreased dramatically at $\mathrm{pH} 6.0$ and was abolished at $\mathrm{pH}$ 6.5. The $\mathrm{pH}$ values of the leaf tissue apoplast range from 5.0 to about 7.0 according to species, cell types and environmental conditions (Husted and Schjoerring 1995, Lopez-Millan et al. 2000, 
Loubet et al. 2002, Muhling and Lauchli 2000, Muhling et al. 1995, Pfanz and Dietz 1987, Pitann et al. 2009). This means that these derivatives may not be taken up by mesophyll cells and marginally loaded in the phloem.

The protonophore CCCP which dissipates the proton motive force is a potent inhibitor of sugar and amino acid uptake activities. Under our experimental conditions, it reduced phloem sap exudation and inhibited almost completely L-13 systemicity (Fig. 6). Therefore, these data indicate that the phloem transport of compound $\mathbf{L - 1 3}$ is governed by a stereospecific amino acid carrier system energized by the transmembrane electrochemical gradient of protons. The proton-motive force is generated by the plasma membrane $\mathrm{H}^{+}$-ATPase, an intrinsic enzyme highly expressed in companion cells and cotyledon epidermal cells (BouchéPillon et al. 1994a, Bouché-Pillon et al. 1994b, DeWitt and Sussman 1995, Morsomme and Boutry 2000). In that respect, Ricinus cotyledon tissues, which generates high transmembrane pH and electrical gradients (Komor et al. 1980), constitute an appropriate model to study the transport properties of xenobiotics.

\section{Conclusion}

A fenpiclonil-glucose and two fenpiclonil-glutamic acid conjugates were synthesized to describe a vectorization strategy for carrier-mediated phloem systemic agrochemical. Because the -NH- function of the pyrrole ring is required for the fungicidal activity (Nyfeler and Ackermann 1992), these compounds are not expected to exhibit a biological activity without releasing the parent compound. Our work must be considered as a new systemic profungicide approach. These large and chlorinated conjugates which violate both Lipinski and Veber rules are found in the phloem sap. However, the fenpiclonil-glutamic acid conjugate (L-13) showed a much more favorable phloem mobility than fenpiclonil-glucose conjugate, indicating that amino acid carriers are more promising than sugar carriers to enhance the phloem loading of phenylpyrrole conjugates. In addition, the phloem mobility of L-glutamic acid conjugate (L13) was about 5-fold higher than that of corresponding D-isomer (D-13), suggesting the involvement of a stereoselective transporter. Further investigation of mechanism displayed that compound $\mathbf{L - 1 3}$ was $\mathrm{pH}-$ dependent and inhibited by the protonophore CCCP. Thus, conjugation of parent pesticides with L-amino acid is a feasible strategy to confer phloem mobility to agrochemicals. The result of the present work about fenpiclonil-amino acid conjugates reinforces the previous conclusion that an amino acid transport system is able to recognize and transport chlorinated xenobiotics of larger size than its natural substrates. In our 
previous paper (Deletage-Grandon, 2001), a halogenated xenobiotic (approximately 350 D) with an amino acid moiety (Lys-2.4 D) was proved to be recognized and translocated by an amino acid transport system. The distribution study of conjugate Lys-2.4 D and parent compound (2.4-D) showed a significantly different accumulation in certain plant organs. The parent molecule (2.4-D) is a phloem mobile herbicide, but the accumulation of Lys-2.4D in the root system is 5 to 10 times greater than that of 2,4D. Therefore, the distribution pattern of amino acid conjugates of fungicides in the whole plant deserves further investigation.

\section{Acknowledgments}

The authors are grateful to FranceAgriMer, InterLoire and Jas Hennessy \& Co. for their financial support of this work. This work was also supported by China Scholarship Council (Hanxiang Wu grant for his PhD). We would like to thank Emilie Dugaro for his kind collaboration in organic synthesis and Christine Bonnemain and Dr Rémi Lemoine for their help to improve the manuscript.

\section{References}

Albert A (1958) Chemical aspects of selective toxicity. Nature. 182: 421-422

Avram S, Funar-Timofei S, Borota A, Chennamaneni SR, Manchala AK, Muresan S (2014) Quantitative estimation of pesticide-likeness for agrochemical discovery. J. Cheminform. 6: 11 doi: 10.1186/s13321-014-0042-6

Barlow CA, Randolph PA (1978) Quality and quantity of plant sap available to pea aphid (Acyrthosiphon pisum Harris aphididae). Ann. Entomol. Soc. Am. 71: 46-48

Bonnemain JL (2010) Aphids as biological models and agricultural pests. C. R. Biol. 333: 461-463 doi: 10.1016/j.crvi.2010.04.002

Bouché-Pillon S, Fleurat-Lessard P, Fromont JC, Serrano R, Bonnemain JL (1994a) Immunolocalization of the plasma membrane H+-ATPase in minor veins of Vicia faba in relation to phloem loading. Plant Physiol. 105: 691-697

Bouché-Pillon S, Fleurat-Lessard P, Serrano R, Bonnemain JL (1994b) Asymmetric distribution of the plasma membrane H+-ATPase in embryos of Vicia faba L with special reference to transfer cells. Planta. 193: 392-397

Cagnoni AJ, Varela O, Gouin SG, Kovensky J, Uhrig ML (2011) Synthesis of multivalent glycoclusters from 1-thio-beta-D-galactose and their inhibitory activity against the beta-galactosidase from E. coli. J. Org. Chem. 76: 3064-3077 doi: 10.1021/jo102421e

Chollet J-F, Couderchet M, Bonnemain J-L (2014) Crop protection: new strategies for sustainable development. Environ. Sci. Pollut. Res. Int. 21: 4793-4796 doi: 10.1007/s11356-014-2567-7

Chollet JF, Deletage C, Faucher M, Miginiac L, Bonnemain JL (1997) Synthesis and structure-activity relationships of some pesticides with an alpha-amino acid function. Biochim. Biophys. Acta. 1336: 331-341 doi: 10.1016/s0304-4165(97)00041-x 
Chollet JF, Rocher F, Jousse C, Deletage-Grandon C, Bashiardes G, Bonnemain JL (2004) Synthesis and phloem mobility of acidic derivatives of the fungicide fenpiclonil. Pest Manage. Sci. 60: 1063-1072 doi: 10.1002/ps.906

Chollet JF, Rocher F, Jousse C, Deletage-Grandon C, Bashiardes G, Bonnemain JL (2005) Acidic derivatives of the fungicide fenpiclonil: effect of adding a methyl group to the $\mathrm{N}$-substituted chain on systemicity and fungicidal activity. Pest Manage. Sci. 61: 377382 doi: $10.1002 /$ ps.977

Dedryver CA, Le Ralec A, Fabre F (2010) The conflicting relationships between aphids and men: A review of aphid damage and control strategies. C. R. Biol. 333: $539-553$ doi: 10.1016/j.crvi.2010.03.009

DeGorter MK, Xia CQ, Yang JJ, Kim RB (2012) Drug transporters in drug efficacy and toxicity. Annu. Rev. Pharmacol. Toxicol. 52: 249-273 doi: 10.1146/annurevpharmtox-010611-134529

Delétage-Grandon C, Chollet JF, Faucher M, Rocher F, Komor E, Bonnemain JL (2001) Carrier-mediated uptake and phloem systemy of a 350-Dalton chlorinated xenobiotic with an alpha-amino acid function. Plant Physiol. 125: 1620-1632 doi: $10.1104 /$ pp.125.4.1620

Despeghel JP, Delrot S (1983) Energetics of amino acid uptake by Vicia faba leaf tissues. Plant Physiol. 71: 1-6 doi: 10.1104/pp.71.1.1

DeWitt ND, Sussman MR (1995) Immunocytological localization of an epitope-tagged plasma membrane proton pump (H+-ATPase) in phloem companion cells. Plant Cell. 7: 2053-2067

Dinant S, Bonnemain JL, Girousse C, Kehr J (2010) Phloem sap intricacy and interplay with aphid feeding. C. R. Biol. 333: 504-515 doi: 10.1016/j.crvi.2010.03.008

Dogimont C, Bendahmane A, Chovelon V, Boissot N (2010) Host plant resistance to aphids in cultivated crops: Genetic and molecular bases, and interactions with aphid populations. C. R. Biol. 333: 566-573 doi: 10.1016/j.crvi.2010.04.003

Dufaud A, Chollet JF, Rudelle J, Miginiac L, Bonnemain JL (1994) Derivatives of pesticides with an alpha-amino acid function - Synthesis and effect on threonine uptake. Pestic. Sci. 41: 297-304 doi: 10.1002/ps.2780410403

Edgington LV (1981) Structural requirements of systemic fungicides. Annu. Rev. Phytopathol. 19: 107-124 doi: 10.1146/annurev.py.19.090181.000543

Fan W, Wu Y, Li XK, Yao N, Li X, Yu YG, Hai L (2011) Design, synthesis and biological evaluation of brain-specific glucosyl thiamine disulfide prodrugs of naproxen. Eur. J. Med. Chem. 46: 3651-3661 doi: 10.1016/j.ejmech.2011.05.029

Frommer WB, Kwart M, Hirner B, Fischer WN, Hummel S, Ninnemann O (1994) Transporters for nitrogenous compounds in plants. Plant Mol. Biol. 26: 1651-1670 doi: $10.1007 / \mathrm{bf} 00016495$

Giacomini KM et al. (2010) Membrane transporters in drug development. Nat. Rev. Drug Discov. 9: 215-236 doi: 10.1038/nrd3028

Girousse C, Bonnemain JL, Delrot S, Bournoville R (1991) Sugar and amino acid composition of phloem sap of Medicago sativa - A comparative study of 2 collecting methods. Plant Physiol. Biochem. 29: 41-48

Gynther M, Laine K, Ropponen J, Leppanen J, Mannila A, Nevalainen T, Savolainen J, Jarvinen TT, Rautio J (2008) Large neutral amino acid transporter enables brain drug delivery via prodrugs. J. Med. Chem. 51: 932-936 doi: 10.1021/jm701175d

Hayashi H, Chino M (1986) Collection of pure phloem sap from wheat and its chemical composition. Plant Cell Physiol. 27: 1387-1393 
Hu AL, Yang W, Xu HH (2010) Novel fluorescent conjugate containing glucose and NBD and its carrier-mediated uptake by tobacco cells. J. Photochem. Photobiol., B. 101: 215-223 doi: 10.1016/j.jphotobiol.2010.07.006

Huisgen R, Knorr R, Mobius L, Szeimies G (1965) 1.3-Dipolare cycloadditionen. 23. Einige beobachtungen zur addition organischer azide an cc-dreifachbindungen. Chem. Ber. Recl. 98: 4014-4021 doi: 10.1002/cber.19650981228

Husted S, Schjoerring JK (1995) Apoplastic $\mathrm{pH}$ and ammonium concentration in leaves of Brassica napus L. Plant Physiol. 109: 1453-1460

Kallarackal J, Komor E (1989) Transport of hexoses by the phloem of Ricinus communis L seedlings. Planta. 177: 336-341 doi: 10.1007/bf00403591

Kolb HC, Finn MG, Sharpless KB (2001) Click chemistry: diverse chemical function from a few good reactions. Angew. Chem. Int. Ed. 40: 2004-2021 doi: 10.1002/15213773(20010601)40:11<2004::aid-anie2004>3.0.co;2-5

Komor E, Rotter M, Waldhauser J, Martin E, Cho BH (1980) Sucrose proton symport for phloem loading in the Ricinus seedling. Ber. Dtsch. bot. Ges. 93: 211-219

Li ZC, Bush DR (1992) Structural determinants in substrate recognition by proton amino acid symports in plasma membrane vesicles isolated from sugar beet leaves. Arch. Biochem. Biophys. 294: 519-526 doi: 10.1016/0003-9861(92)90719-d

Lipinski CA, Lombardo F, Dominy BW, Feeney PJ (2012) Experimental and computational approaches to estimate solubility and permeability in drug discovery and development settings. Adv. Drug Del. Rev. 64: 4-17 doi: 10.1016/j.addr.2012.09.019

Liu DD, Chao WM, Turgeon R (2012) Transport of sucrose, not hexose, in the phloem. J. Exp. Bot. 63: 4315-4320 doi: 10.1093/jxb/ers127

Lopez-Millan AF, Morales F, Abadia A, Abadia J (2000) Effects of iron deficiency on the composition of the leaf apoplastic fluid and xylem sap in sugar beet. Implications for iron and carbon transport. Plant Physiol. 124: 873-884 doi: 10.1104/pp.124.2.873

Loubet B, Milford C, Hill PW, Tang YS, Cellier P, Sutton MA (2002) Seasonal variability of apoplastic $\mathrm{NH} 4+$ and $\mathrm{pH}$ in an intensively managed grassland. Plant Soil. 238: 97-110 doi: 10.1023/a:1014208926195

Mannhold R, Kubinyi H, Folkers G, van de Waterbeemd H, Testa B (Editors), 2009: Drug bioavailability: estimation of solubility, permeability, absorption and bioavailability, 40. John Wiley \& Sons, Weinheim

Mayer T, Maier ME (2007) Design and synthesis of a tag-free chemical probe for photoaffinity labeling. Eur. J. Org. Chem.: 4711-4720 doi: 10.1002/ejoc.200700188

Morsomme P, Boutry M (2000) The plant plasma membrane H+-ATPase: structure, function and regulation. Biochim. Biophys. Acta. 1465: 1-16 doi: 10.1016/s00052736(00)00128-0

Muhling KH, Plieth C, Hansen UP, Sattelmacher B (1995) Apoplastic pH of intact leaves of Vicia faba as influenced by light. J. Exp. Bot. 46: 377-382 doi: 10.1093/jxb/46.4.377

Muhling KH, Lauchli A (2000) Light-induced $\mathrm{pH}$ and $\mathrm{K}+$ changes in the apoplast of intact leaves. Planta. 212: 9-15 doi: 10.1007/s004250000374

Nyfeler R, Ackermann P (1992) Phenylpyrroles, a new class of agricultural fungicides related to the natural antibiotic pyrrolnitrin. ACS Symp. Ser. 504: 395-404

Pfanz H, Dietz KJ (1987) A fluorescence method for the determination of the apoplastic proton concentration in intact leaf tissues. J. Plant Physiol. 129: 41-48

Pitann B, Schubert S, Muhling KH (2009) Decline in leaf growth under salt stress is due to an inhibition of $\mathrm{H}+$-pumping activity and increase in apoplastic $\mathrm{pH}$ of maize leaves. J. Plant Nutr. Soil Sci. 172: 535-543 doi: 10.1002/jpln.200800349 
Rocher F, Chollet JF, Jousse C, Bonnemain JL (2006) Salicylic acid, an ambimobile molecule exhibiting a high ability to accumulate in the phloem. Plant Physiol. 141: 1684-1693 doi: $10.1104 / p p .106 .082537$

Rocher F, Chollet JF, Legros S, Jousse C, Lemoine R, Faucher M, Bush DR, Bonnemain JL (2009) Salicylic acid transport in Ricinus communis involves a pH-dependent carrier system in addition to diffusion. Plant Physiol. 150: 2081-2091 doi: 10.1104/pp.109.140095

Rocher F, Roblin G, Chollet J-F (2016) Modifications of the chemical structure of phenolics differentially affect physiological activities in pulvinar cells of Mimosa pudica L. II. Influence of various molecular properties in relation to membrane transport. Environ. Sci. Pollut. Res. Int.: In press doi: 10.1007/s11356-016-6048-Z

Rostovtsev VV, Green LG, Fokin VV, Sharpless KB (2002) A stepwise Huisgen cycloaddition process: Copper(I)-catalyzed regioselective "ligation" of azides and terminal alkynes. Angew. Chem. Int. Ed. 41: 2596-2599 doi: 10.1002/15213773(20020715)41:14<2596::aid-anie2596>3.0.co;2-4

Sheehan JC, Hess GP (1955) A new method of forming peptide bonds. J. Am. Chem. Soc. 77: 1067-1068 doi: 10.1021/ja01609a099

Sheehan JC, Boshart GL, Cruickshank PA (1961) Convenient synthesis of water-soluble carbodiimides. J. Org. Chem. 26: 2525-2528 doi: 10.1021/jo01351a600

Siebum AHG, Tsang RKF, van der Steen R, Raap J, Lugtenburg J (2004) Synthesis of (epsilon-C-13-,epsilon N-15)-enriched L-lysine - Establishing schemes for the preparation of all possible C-13 and N-15 isotopomers of L-lysine, L-ornithine, and Lproline. Eur. J. Org. Chem.: 4391-4396 doi: 10.1002/ejoc.200400370

Stella VJ, Borchardt RT, Hageman MJ, Oliyai R, Maag H, Tilley JW (Editors), 2007: Prodrugs challenges and rewards Part 1. Springer-Verlag, New York, XVIII, 1464 pp

Sugano K, Kansy M, Artursson P, Avdeef A, Bendels S, Di L, Ecker GF, Faller B, Fischer H, Gerebtzoff G, Lennernaes H, Senner F (2010) Coexistence of passive and carriermediated processes in drug transport. Nat. Rev. Drug Discov. 9: 597-614 doi: $10.1038 / \mathrm{nrd} 3187$

Tice CM (2001) Selecting the right compounds for screening: does Lipinski's Rule of 5 for pharmaceuticals apply to agrochemicals? Pest Manage. Sci. 57: 3-16 doi: 10.1002/1526-4998(200101)57:1<3::aid-ps269>3.0.co;2-6

Tornoe CW, Christensen C, Meldal M (2002) Peptidotriazoles on solid phase: 1,2,3 -triazoles by regiospecific copper(I)-catalyzed 1,3-dipolar cycloadditions of terminal alkynes to azides. J. Org. Chem. 67: 3057-3064 doi: 10.1021/jo011148j

Usuki T, Sugimura T, Komatsu A, Koseki Y (2014) Biomimetic chichibabin pyridine synthesis of the COPD biomarkers and elastin cross-linkers isodesmosine and desmosine. Org. Lett. 16: 1672-1675 doi: 10.1021/ol500333t

van Pee KH, Ligon JM (2000) Biosynthesis of pyrrolnitrin and other phenylpyrrole derivatives by bacteria. Nat. Prod. Rep. 17: 157-164 doi: 10.1039/a902138h

Veber DF, Johnson SR, Cheng HY, Smith BR, Ward KW, Kopple KD (2002) Molecular properties that influence the oral bioavailability of drug candidates. J. Med. Chem. 45: 2615-2623 doi: 10.1021/jm020017n

Wang CJ, Liu ZQ (2007) Foliar uptake of pesticides - Present status and future challenge. Pestic. Biochem. Physiol. 87: 1-8 doi: 10.1016/j.pestbp.2006.04.004

Wu HX, Yang W, Zhang ZX, Huang T, Yao GK, Xu HH (2012) Uptake and phloem transport of glucose-fipronil conjugate in Ricinus communis involve a carrier-mediated mechanism. J. Agric. Food Chem. 60: 6088-94 doi: 10.1021/jf300546t

Yang W, Wu HX, Xu HH, Hu AL, Lu ML (2011) Synthesis of glucose-fipronil conjugate and its phloem mobility. J. Agric. Food Chem. 59: 12534-12542 doi: 10.1021/jf2031154 
Zawilska JB, Wojcieszak J, Olejniczak AB (2013) Prodrugs: a challenge for the drug development. Pharmacol. Rep. 65: 1-14 


\section{Figure legends}

Figure 1. General reaction scheme showing the different steps of the synthesis of the sugarfungicide or amino acid- fungicide derivatives. DMAP: 4-Dimethylaminopyridine; EDCl: 1-Ethyl3-(3-dimethylaminopropyl)carbodiimide hydrochloride; TFA: Trifluoroacetic acid

Figure 2. Azido and fenpiclonil derivatives numbering for ${ }^{1} \mathrm{H}$ and ${ }^{13} \mathrm{C}$ assignments

Figure 3. Time course of amino acid conjugates L-13 (A) and D-13 (B) concentration in phloem sap of Ricinus. Cotyledons were incubated in a standard buffered solution at $\mathrm{pH} 5.0$ (Rocher et al, 2006) for $30 \mathrm{~min}$, then in the same solution containing compounds L-13 or D-13 at $100 \mu \mathrm{M}$ concentration. After $30 \mathrm{~min}$, the hypocotyl was severed in the hook region and then the sap was collected every hour for $6 \mathrm{~h}$. For each time, the Mann-Whitney test was used to assess statistically significant differences between the two sets at the $5 \%$ probability level. Except for $\mathrm{t}=1 \mathrm{~h}$ where $\mathrm{p}$ value $=0.006$, all $\mathrm{p}$-values $<0.0001$. For box plots, $\mathrm{n}=22(\mathrm{~A}), \mathrm{n}=10(\mathrm{~B})$.

Figure 4. Time course of glucose conjugate $\mathbf{1 2}$ concentration in phloem sap of Ricinus. Cotyledons were incubated in a standard buffered solution at pH 5.0 (Rocher et al, 2006) for 30 min, then in the same solution containing compound 12 at $100 \mu \mathrm{M}$ (A) or $500 \mu \mathrm{M}$ (B)

concentration. After $30 \mathrm{~min}$, the hypocotyl was severed in the hook region and then the sap was collected every hour for $6 \mathrm{~h}$. For box plots, $\mathrm{n}=20$.

Figure 5. Concentration (A) or quantity exported (B) of compound L-13 in phloem sap of Ricinus as a function of the $\mathrm{pH}$ of the incubation medium. Cotyledons were incubated in a standard buffered solution at $\mathrm{pH}$ 5.0, 6.0, 7.0 or 8.0 (Rocher et al, 2006) for $30 \mathrm{~min}$, then in the same solution containing compound L-13 at $100 \mu \mathrm{M}$ concentration. After $30 \mathrm{~min}$, the hypocotyl was severed in the hook region and then the sap was collected between the fourth and fifth hour. The Kruskal-Wallis test was used to assess statistically significant differences between the different sets at the $5 \%$ probability level. A different letter indicate that the sets are not from the same population. For box plots, $\mathrm{n}=20$.

Figure 6. Effect of CCCP on time course of conjugate L-13 concentration (A) or quantity exported (B) in phloem sap of Ricinus. Cotyledons were incubated in a standard buffered solution at $\mathrm{pH} 5.0$ (Rocher et al, 2006) for $30 \mathrm{~min}$, then in the same solution containing compound $\mathbf{L}-\mathbf{1 3}$ at $100 \mu \mathrm{M}$ concentration without (C : Control) or with $\mathrm{CCCP}$ at $10 \mu \mathrm{M}$ final concentration. After 30 min, the hypocotyl was severed in the hook region and then the sap was collected every hour for 3 h. For each time, the Mann-Whitney test was used to assess statistically significant differences between the two sets (without or with CCCP) at the $5 \%$ probability level. In all cases, p-values < 0.0001. For box plots, $\mathrm{n}=24$ (control), $\mathrm{n}=19$ (CCCP). 